\title{
The Navier-Stokes equations on the plane with time-dependent external forces
}

\section{Masao Yamazaki ${ }^{1}$}

Published online: 13 July 2021

(c) The Author(s) 2021

\begin{abstract}
We are concerned with the non-stationary Navier-Stokes equations on the whole plane with external forces which are non-decaying in time, and give a sufficient condition on the external forces for the existence of a solution which exists for whole time. Typical examples are timeperiodic solutions and solutions almost periodic in time. The stability under perturbation is also verified.
\end{abstract}

Keywords Navier-Stokes equation - Time-periodic function - Almost periodic function . Weighted Lorentz spaces

Mathematics Subject Classification 35Q30

\section{Introduction}

We are concerned with the non-stationary Navier-Stokes equations on the whole plane

$$
\begin{aligned}
\frac{\partial u}{\partial t}(x, t)-\Delta_{x} u(x, t)+(u(x, t) \cdot \nabla) u(x, t)+\nabla \pi(x, t) & =f(x, t), \\
\nabla \cdot u(x, t) & =0
\end{aligned}
$$

for $x \in \mathbb{R}^{2}$ and $t \in \mathbb{R}$, where $u(x, t)=\left(u_{1}(x, t), u_{2}(x, t)\right)$ is a vector-valued unknown function standing for the velocity, $\pi(x, t)$ is a scalar-valued function standing for the pressure, and $f(x, t)=\left(f_{1}(x, t), f_{2}(x, t)\right)$ is a given function standing for the external force.

Partly supported by the International Research Training Group (IGK 1529) on Mathematical Fluid Dynamics funded by DFG and JSPS and associated with TU Darmstadt, Waseda University and the University of Tokyo, and by Grant-in-Aid for Scientific Research (C) 17K05339, Ministry of Education, Culture, Sports, Science and Technology, Japan.

This article is part of the topical collection dedicated to Prof. Hideo Kozono on the occasion of his 60th birthday, edited by Kazuhiro Ishige, Tohru Ozawa, Senjo Shimizu, and Yasushi Taniuchi.

\footnotetext{
$\bowtie \quad$ Masao Yamazaki

masao.yamazaki@waseda.jp

1 Waseda University, Okubo 3-chome, Shinjuku, Tokyo 169-8555, Japan
} 
More precisely, we consider two cases. One is to consider the system $(0.1)-(0.2)$ for $(x, t) \in \mathbb{R}^{2} \times \mathbb{R}$; namely, we consider the external force is defined for all $t \in \mathbb{R}$. Typical examples are time-periodic functions or almost periodic functions in time. The other one is the Cauchy problem; namely, we consider (0.1)-(0.2) for $(x, t) \in \mathbb{R}^{2} \times(0, \infty)$, together with the initial condition

$$
u(x, 0)=u_{0}(x) \text { on } \mathbb{R}^{2}
$$

with a function $\nabla \cdot u_{0}(x)=0$. In particular, we are concerned with the existence and uniqueness in the small, together with the stability under small initial perturbations, for small external force (and initial datum) in appropriate classes. Throughout this paper we assume that $f(x, t)$ is given by the potential functions $F_{j, k}(x, t)$ with the formula

$$
f_{j}(x, t)=\sum_{k=1}^{2} \frac{\partial F_{j, k}}{\partial x_{k}}(x, t) \text { for } j=1,2,
$$

namely,

$$
\nabla \cdot F(x, t)=\left(\begin{array}{l}
\frac{\partial F_{1,1}}{\partial x_{1}}(x, t)+\frac{\partial F_{1,2}}{\partial x_{2}}(x, t) \\
\frac{\partial F_{2,1}}{\partial x_{1}}(x, t)+\frac{\partial F_{2,2}}{\partial x_{2}}(x, t)
\end{array}\right) .
$$

Maremonti [18,19] first considered problems of this type on unbounded domains. Then Salvi [25], Kozono and Nakao [14], Maremonti and Padula [20], the author [27], Galdi and Sohr [10], Kubo [15], Galdi and Silvestre [8,9], Crispo and Maremonti [3], Farwig and Okabe [5], Silvestre [26], Iwabuchi and Takada [11], Nguyen [23], Kozono et al. [13], Kyed [16], Lemarié-Rieusset [17], Farwig et al. [4] considered similar problems, mostly for time-periodic solutions. However, their results are limited to the case where the space dimension is larger than or equal to three. On the other hand, Kobayashi [12] considered the two-dimensional case, but his solutions correspond to weak solutions and the uniqueness is not known. As far as the author knows, only Galdi [6,7] obtained the unique existence for two-dimensional case, but the method relies on the periodicity.

The purpose of this paper is to solve the problems above on the whole plane $\mathbb{R}^{2}$ in the class of critically decreasing solutions or supercritically decreasing functions introduced by the author in $[28,29]$. Then we obtain the unique existence of small solutions, together with the continuous dependence on the datum, in these classes under appropriate assumptions on $F(x, t)$. Our approach does not rely on the periodicity, and hence we can consider almost periodic solutions in time, and more general solutions. We also obtain the stability of this solution by showing the global solvability and show the asymptotic behavior of the Cauchy problems with small initial disturbance.

Since our problems is a generalization of the stationary problems with solutions in the classes above with time-independent external forces, we need to impose some symmetry condition, such as

$$
u\left(x^{\perp}\right)=(u(x))^{\perp},
$$

where $x^{\perp}=\left(-x_{2}, x_{1}\right)$. This condition, introduced in the author [29] for the unique existence of small stationary solution with stationary external force, implies that the solution is equivariant under the action of the cyclic group $C_{4}=\left\{i d, \sigma, \sigma^{2}, \sigma^{3}\right\}$ of order 4 , where $\sigma(x)=x^{\perp}$. The viewpoint above is first introduced by Brandolese [2], which is concerned with sharp decay rate of the solution of the Cauchy problems. 
In the critical case $u(x)$ decays like $1 /|x|$. Hence, in order to treat the nonlinear terms $u_{1}(x) u_{2}(x)$ and $\left(u_{1}(x)\right)^{2}-\left(u_{2}(x)\right)^{2}$, it seems natural to introduce Hardy space or some modification, as in Miyakawa [21,22] and Okabe and Tsutsui [24]. However, in order to apply the fixed point argument, we need to keep symmetry, and hence we cannot rely merely on the theory of Hardy spaces. Hence we employ weighted $L^{p}$-space and show the weighted estimates of the Stokes semigroup instead, by regarding the Gauss kernel as a singular integral and applying its boundedness on weighted spaces.

Moreover, particularly in the treatment of critically decreasing functions, we need to consider improper integrals which is not Bochner integrable. We get around this difficulty by showing the convergence in the weak-* topology, which need to consider the dual problem and our solutions are obtained as very weak solutions, as is done in [27]. However, it is not clear whether the solutions thus obtained are strong solutions or not. Hence we start with the study of strong solutions of linear problems. Moreover, to employ this argument we need to consider the Lorentz spaces in place of the standard $L^{p}$-spaces.

In order to state the assumption on $F(x, t)$, we introduce the functions

$$
\begin{array}{ll}
G_{0}[F](x, t)=\frac{F_{1,1}(x, t)+F_{2,2}(x, t)}{2}, & G_{1}[F](x, t)=\frac{F_{1,1}(x, t)-F_{2,2}(x, t)}{2}, \\
G_{2}[F](x, t)=\frac{F_{2,1}(x, t)+F_{1,2}(x, t)}{2}, & G_{3}[F](x, t)=\frac{F_{2,1}(x, t)-F_{1,2}(x, t)}{2},
\end{array}
$$

and impose the conditions

$$
G_{k}[F]\left(x^{\perp}, t\right)=-G_{k}[F](x, t) \text { for } k=1,2 \text { and every } x \in \mathbb{R}^{2}, t \in \mathbb{R}
$$

and

$$
G_{3}[F]\left(x^{\perp}, t\right)=G_{3}[F](x, t) \text { for every } x \in \mathbb{R}^{2}, t \in \mathbb{R} \text {. }
$$

The outline of this paper is as follows. In Sect. 1 we rewrite the system, fix notations and state the results, Next we reduce the problems into integral equations in Sect. 2. Then we introduce linear estimate in Sect. 3, Finally in Sect. 4 we prove the theorems in Sect. 2.

\section{Results}

We first rewrite the system in order to state the assumptions. Since $\nabla \cdot u(x, t)=0$, we can write

$$
(u(x, t) \cdot \nabla) u_{j}(x, t)=\sum_{k=1}^{2} \frac{\partial}{\partial x_{k}}\left(u_{k}(x, t) u_{j}(x, t)\right) \text { for } j=1,2
$$

Substituting these equalities and (0.4) into (0.1), we obtain

$$
\frac{\partial u}{\partial t}(x, t)-\Delta_{x} u(x, t)+\nabla \pi(x, t)=\nabla \cdot \tilde{F}[u](x, t),
$$


where $\tilde{F}_{j, k}[u](x, t)=F_{j, k}(x, t)-u_{j}(x, t) u_{k}(x, t)$ for $j, k=1,2$. We next put

$$
\begin{aligned}
& H_{0}[F, u](x, t)=\frac{\tilde{F}_{1,1}[u](x, t)+\tilde{F}_{2,2}[u](x, t)}{2}, \\
& H_{1}[F, u](x, t)=\frac{\tilde{F}_{1,1}[u](x, t)-\tilde{F}_{2,2}[u](x, t)}{2}, \\
& H_{2}[F, u](x, t)=\frac{\tilde{F}_{2,1}[u](x, t)+\tilde{F}_{1,2}[u](x, t)}{2}, \\
& H_{3}[F, u](x, t)=\frac{\tilde{F}_{2,1}[u](x, t)-\tilde{F}_{1,2}[u](x, t)}{2} .
\end{aligned}
$$

Then we have $H_{k}[F, u](x, t)=G_{k}[F](x, t)+H_{k}[0, u](x, t)$ for every $k=0,1,2,3$, and $H_{3}[0, u]=0$ holds for every $u(x, t)$. It follows that $H_{3}[F, u](x, t)=G_{3}[F](x, t)$, Moreover, we have the equalities

$$
\begin{aligned}
& \tilde{F}_{1,1}[u](x, t)=H_{0}[F, u](x, t)+H_{1}[F, u](x, t), \\
& \tilde{F}_{2,2}[u](x, t)=H_{0}[F, u](x, t)-H_{1}[F, u](x, t), \\
& \tilde{F}_{1,2}[u](x, t)=H_{2}[F, u](x, t)-G_{3}[F](x, t), \\
& \tilde{F}_{2,1}[u](x, t)=H_{2}[F, u](x, t)+G_{3}[F](x, t) .
\end{aligned}
$$

Substituting these equalities into (1.1) we have

$$
\begin{aligned}
& \frac{\partial u_{1}}{\partial t}(x, t)-\Delta_{x} u_{1}(x, t)+\frac{\partial \tilde{\pi}}{\partial x_{1}}(x, t) \\
& \quad=\frac{\partial H_{1}[F, u]}{\partial x_{1}}(x, t)+\frac{\partial H_{2}[F, u]}{\partial x_{2}}(x, t)-\frac{\partial G_{3}[F]}{\partial x_{2}}(x, t), \\
& \frac{\partial u_{2}}{\partial t}(x, t)-\Delta_{x} u_{2}(x, t)+\frac{\partial \tilde{\pi}}{\partial x_{2}}(x, t) \\
& \quad=-\frac{\partial H_{1}[F, u]}{\partial x_{2}}(x, t)+\frac{\partial H_{2}[F, u]}{\partial x_{1}}(x, t)+\frac{\partial G_{3}[F]}{\partial x_{1}}(x, t),
\end{aligned}
$$

where $\tilde{\pi}(x, t)=\pi(x, t)-H_{0}[F, u](x, t)$. Thus our problems are to find the solution (1.2)(1.3), together with $\nabla \cdot u(x, t)=0$ on $\mathbb{R}^{2}$, for $t \in \mathbb{R}$ or $t \in(0, \infty)$ with $(0.3)$. In order to remove $\tilde{\pi}(x, t)$, we introduce the Helmholtz projection $P$ defined by the matrix $\left(\delta_{j, k}+R_{j} R_{k}\right)$, where $R_{j}$ denotes the Riesz operator. Then we can rewrite (1.2)-(1.3) into

$$
=P\left(\begin{array}{c}
\frac{\partial u}{\partial t}(x, t)-\Delta u(x, t) \\
\frac{\partial H_{1}[F, u]}{\partial x_{1}}(x, t)+\frac{\partial H_{2}[F, u]}{\partial x_{2}}(x, t)-\frac{\partial G_{3}[F]}{\partial x_{2}}(x, t) \\
-\frac{\partial H_{1}[F, u]}{\partial x_{2}}(x, t)+\frac{\partial H_{2}[F, u]}{\partial x_{1}}(x, t)+\frac{\partial G_{3}[F]}{\partial x_{1}}(x, t)
\end{array}\right) .
$$

In order to treat the critical case, we introduce the Lorentz space. Suppose that $K$ is a Lebesgue measurable subset of $\mathbb{R}^{n}$ and $f(x)$ is a Lebesgue measurable function on $K$. Then we put

$$
f^{*}(t)=\sup \{s \in(0, \infty) \mid \mu(\{x \in K|| f(x) \mid>s\})>t\}
$$


for every $t>0$. Then, for $0<p<\infty$ and $0<\rho \leq \infty$, the Lorentz space $L^{p, \rho}(K)$ is defined as $L^{p, \rho}(K)=\left\{f(x) \mid\|f\|_{p, \rho}<\infty\right\}$, where

$$
\|f\|_{p, \rho}= \begin{cases}\left(\int_{0}^{\infty}\left(t^{1 / p} f^{*}(t)\right)^{\rho} \frac{d t}{t}\right)^{1 / \rho} & \text { for } 0<\rho<\infty, \\ \sup _{t>0} t^{1 / p} f^{*}(t) & \text { for } \rho=\infty\end{cases}
$$

For detailed properties of the Lorentz spaces and real interpolation, see Bergh and Löfström [1] for example. In the following we list the properties needed in this paper. For $p \in[1, \infty)$, the space $L^{p, p}(K)$ coincides with the standard Lebesgue space $L^{p}(K)$ equipped with the norm $\|f\|_{p}$. In general, the function $\|f\|_{p, \rho}$ does not satisfy the triangle inequality. However, if $f \in L^{p, q}(K)$ with $1<p<\infty$ and $1 \leq \rho \leq \infty$, the function $f^{* *}(t)=\frac{1}{t} \int_{0}^{t} f^{*}(s) d s$ is well-defined for every $t>0$, and the functional

$$
\|f\|_{p, \rho}^{*}= \begin{cases}\left(\int_{0}^{\infty}\left(t^{1 / p} f^{* *}(t)\right)^{\rho} \frac{d t}{t}\right)^{1 / \rho} & \text { for } 0<\rho<\infty \\ \sup _{t>0} t^{1 / p} f^{* *}(t) & \text { for } \rho=\infty\end{cases}
$$

satisfies the triangle inequality and the estimate

$$
\|f\|_{p, \rho} \leq\|f\|_{p, \rho}^{*} \leq \frac{p}{p-1}\|f\|_{p, \rho} .
$$

Hence $L^{p, \rho}(K)$ becomes a Banach space with norm $\|f\|_{p, \rho}^{*}$. Further, let $L^{p, \infty-}(K)$ denote the closure of $L^{p}(K)$ in $L^{p, \infty}(K)$. Then, for $p \in(1, \infty)$ and $1 \leq \rho<\infty$ or $\rho=\infty$, the space $L^{p, \rho}(K)$ is the dual space of $L^{p /(p-1), \rho^{\prime}}(K)$, where

$$
\rho^{\prime}= \begin{cases}\frac{\infty-}{\rho} & \text { if } \rho=1 \\ \frac{\rho-1}{\rho-1} & \text { if } 1<\rho<\infty \\ 1 & \text { if } \rho=\infty\end{cases}
$$

Another important property is the real interpolation: Suppose that $p_{0}, p_{1} \in(0, \infty)$, $\rho_{0}, \rho_{1}, \rho \in(0, \infty], 0<\theta<1$ and $p_{0} \neq p_{1}$. Define $p$ by $1 / p=(1-\theta) / p_{0}+\theta / p_{1}$. Then the real interpolation space $\left(L^{p_{0}, \rho_{0}}(K), L^{p_{1}, \rho_{1}}(K)\right)_{\theta, \rho}$ coincides with the Lorentz space $L^{p, \rho}(K)$.

Moreover, we introduce the class of solenoidal vector fields. Let $C_{0, \sigma}^{\infty}\left(\mathbb{R}^{2}\right)$ denote the class of functions $\varphi(x) \in\left(C_{0}^{\infty}\left(\mathbb{R}^{2}\right)\right)^{2}$ such that $\nabla \cdot \varphi(x)=0$. For $p \in(1, \infty)$, the class $L_{\sigma}^{p}\left(\mathbb{R}^{2}\right)$ denote the closure of $C_{0, \sigma}^{\infty}(\Omega)$ in $\left(L^{p}(\Omega)\right)^{2}$. Finally, for $p \in(1, \infty)$ and $\rho \in[1, \infty]$, let $L_{\sigma}^{p, \rho}\left(\mathbb{R}^{2}\right)$ denote the interpolation space $\left(L_{\sigma}^{p_{0}}\left(\mathbb{R}^{2}\right), L_{\sigma}^{p_{1}}\left(\mathbb{R}^{2}\right)\right)_{\theta, \rho}$, where $p_{0}, p_{1} \in(1, \infty)$, $p_{0} \neq p_{1}, 0<\theta<1$ and $1 / p=(1-\theta) / p_{0}+\theta / p_{1}$. This space is well-defined independent of the choice of $p_{0}$ and $p_{1}$ up to equivalent norms.

We next introduce the class of potential functions and solutions. Suppose that $2<$ $p<4$ and that $1-2 / p \leq a<1 / 2$. We consider external forces $F(x, t)$ satisfying $\langle\cdot\rangle^{2 a} F(\cdot, t) \in B C\left(I,\left(L^{p / 2, \rho}\left(\mathbb{R}^{2}\right)\right)^{4}\right)$, and consider the solution $u(x, t)$ satisfying $\langle\cdot\rangle^{a} u(\cdot, t) \in B C\left(I,\left(L^{p, \rho}\left(\mathbb{R}^{2}\right)\right)^{2}\right)$, where $\langle x\rangle=\left(1+|x|^{2}\right)^{1 / 2}$, and $I=\mathbb{R}$ or $I=(0, \infty)$. 
Since $\langle\cdot\rangle^{-a} \in L^{2 / a, \infty} \cap L^{\infty}$, it follows that the function $u(x, t)$ in the class above satisfies $B C\left(I,\left(L^{2 p /(2+a p), \rho}\left(\mathbb{R}^{2}\right) \cap L^{p, \rho}\left(\mathbb{R}^{2}\right)\right)^{2}\right)$. We thus see that $u(\cdot, t) \in B C\left(I,\left(L^{2,1}\left(\mathbb{R}^{2}\right)\right)^{2}\right)$ if $1-2 / p<a<1 / 2$, and that $u(\cdot, t) \in B C\left(I,\left(L^{2, \rho}\left(\mathbb{R}^{2}\right)\right)^{2}\right)$ if $a=1-2 / p$.

Then our main theorem on the whole time is the following.

Theorem 1.1 Suppose that $2<p<4$ and that either $1-2 / p<a<1 / 2,1 \leq \rho \leq \infty$ or $a=1-2 / p, \rho=\infty$. Then there exist a positive constant $\varepsilon_{0}$ and a strictly monotoneincreasing function $\omega(s)$ on $\left[0, \varepsilon_{0}\right]$ such that $\omega(0)=0$ and that the following assertion holds. Suppose that the function $G[F](x, t)=\left(G_{1}[F](x, t), G_{2}[F](x, t), G_{3}[F](x, t)\right)$ satisfies $\langle\cdot\rangle^{2 a} G[F](\cdot, t) \in B C\left(\mathbb{R},\left(L^{p / 2, \rho}\left(\mathbb{R}^{2}\right)\right)^{3}\right)$ with the estimate

$$
\sup _{t \in \mathbb{R}}\left\|\langle\cdot\rangle^{2 a} G[F](\cdot, t)\right\|_{p / 2, \rho}=\varepsilon \in\left[0, \varepsilon_{0}\right) .
$$

We moreover assume that $G_{1}[F](\cdot, t), G_{2}[F](\cdot, t)$ satisfy $(0.5)$ and that $G_{3}[F](\cdot, t)$ satisfies (0.6) for every $t \in \mathbb{R}$.

Then there exists a solution $u(\cdot, t)$ of the equation (1.4) in $\mathbb{R}$ in the sense of distributions and the symmetry condition (C4E) for every $t \in \mathbb{R}$ such that

$$
\langle\cdot\rangle^{a} u(x) \in B C\left(\mathbb{R},\left(L^{p, \rho}\left(\mathbb{R}^{2}\right)\right)^{2}\right)
$$

with the estimate

$$
\sup _{t \in \mathbb{R}}\left\|\langle\cdot\rangle^{a} u(\cdot, t)\right\|_{p, \rho} \leq \omega(\varepsilon) .
$$

This solution is the unique solution of (1.4) in $\mathbb{R}$ satisfying the symmetry above and the condition (1.7) with the estimate

$$
\sup _{t \in \mathbb{R}}\left\|\langle\cdot\rangle^{a} u(\cdot, t)\right\|_{p, \rho}<\omega\left(\varepsilon_{0}\right) .
$$

Furthermore, for every $\varepsilon^{\prime} \in\left(0, \varepsilon_{0}\right)$, the mapping $\langle\cdot\rangle^{2 a} G[F](\cdot, t) \mapsto\langle\cdot\rangle^{a} u(\cdot, t)$ is uniformly continuous from the closed ball in $B C\left(\mathbb{R},\left(L^{p / 2, \rho}\left(\mathbb{R}^{2}\right)\right)^{3}\right)$ with center 0 and radius $\varepsilon^{\prime}$ to $B C\left(\mathbb{R},\left(L^{p, \rho}\left(\mathbb{R}^{2}\right)\right)^{2}\right)$.

In order to state an immediate consequence of Theorem 1.1, we recall the definition of almost periodic function. A mapping $f$ from $\mathbb{R}$ to a metric space $X$ is called almost periodic if, for every $\varepsilon>0$, there exists a positive number $L$ such that, for every $t_{0}$ there exists a number $T \in\left[t_{0}, t_{0}+L\right]$ such that $d(f(t), f(t+T))<\varepsilon$ holds for every $t \in \mathbb{R}$. Then we have the following corollary.

Corollary 1.2 Suppose that $p, a, \rho$ and $G[F](x, t)$ be the same as in Theorem 1.1, and that $u(x, t)$ is the solution of (1.4) in $\mathbb{R}$ constructed in Theorem 1.1. Then we have the following assertions.

(i) If $G[F](x, t)$ is periodic with respect to $t$ with period $T$, then $u(x, t)$ is also periodic with respect to $t$ with period $T$.

(ii) If $\langle\cdot\rangle^{2 a} G[F](\cdot, t)$ is almost periodic with respect to $t$ in $\left(L^{p / 2, \rho}\left(\mathbb{R}^{2}\right)\right)^{3}$, then $\langle\cdot\rangle^{a} u(x, t)$ is also periodic with respect to $t$ in $\left(L^{p, \rho}\left(\mathbb{R}^{2}\right)\right)^{2}$. 
We also have the result on the Cauchy problem in the same function space, which implies the stability of the solutions constructed in Theorem 2.3 under small perturbation. In order to state the theorem, let $X$ denote the direct product $\left(L^{p, \rho}\left(\mathbb{R}^{2}\right)\right)^{2} \times B C\left((0, \infty),\left(L^{p / 2, \rho}\left(\mathbb{R}^{2}\right)\right)^{3}\right)$ equipped with the metric

$$
d((u, f),(v, g))=\|u-v\|_{p, \rho}+\sup _{t>0}\|f(t)-g(t)\|_{p / 2, \rho} .
$$

Then our result is the following.

Theorem 1.3 Let $p, a$ and $\rho$ be the same as in Theorem 1.1. Suppose that $u_{0}(x)$ satisfies (C4E) and theorem $\nabla \cdot u_{0}(x)=0$, and that $G_{1}[F](\cdot, t), G_{2}[F](\cdot, t)$ satisfy $(0.5)$ and that $G_{3}[F](\cdot, t)$ satisfies $(0.6)$ for every $t>0$. Furthermore, putting

$$
G[F](x, t)=\left(G_{1}[F](x, t), G_{2}[F](x, t), G_{3}[F](x, t)\right),
$$

we assume that $\left(\langle\cdot\rangle^{a} u_{0}(\cdot),\langle\cdot\rangle^{2 a} G[F](\cdot, t)\right) \in X$ with the estimate

$$
d\left(\left(\langle\cdot\rangle^{a} u_{0}(\cdot),\langle\cdot\rangle^{2 a} G[F](\cdot, t)\right),(0,0)\right)=\varepsilon \in\left[0, \varepsilon_{0}\right) .
$$

Then there exists a solution $u(\cdot, t)$ of $(1.4)$ in $(0, \infty)$ in the sense of distributions satisfying (0.2) in the sense

$$
u(x, t) \rightarrow u_{0}(x) \text { as } t \rightarrow+0 \text { in the sense of distributions }
$$

such that the symmetry condition $(\mathrm{C} 4 \mathrm{E})$ holds for every $t>0$ and that

$$
\langle\cdot\rangle^{a} u(\cdot, t) \in B C\left((0, \infty),\left(L^{p, \rho}\left(\mathbb{R}^{2}\right)\right)^{2}\right)
$$

with the estimate

$$
\sup _{t>0}\left\|\langle\cdot\rangle^{a} u(\cdot, t)\right\|_{p, \rho} \leq \omega(\varepsilon) .
$$

Moreover, if $\langle\cdot\rangle^{a} u_{0}(\cdot) \in\left(L^{p, \tilde{\rho}}\left(\mathbb{R}^{2}\right)\right)^{2}$ as well, where

$$
\tilde{\rho}= \begin{cases}\rho & \text { for } 1 \leq \rho<\infty \\ \infty- & \text { for } \rho=\infty\end{cases}
$$

then we have $\langle\cdot\rangle^{a} u(\cdot, t) \rightarrow\langle\cdot\rangle^{a} u_{0}(\cdot)$ in $\left(L^{p, \rho}\left(\mathbb{R}^{2}\right)\right)^{2}$ as $t \rightarrow 0$.

This solution is the unique solution of $(1.4)$ in $(0, \infty)$ satisfying $(1.11)$, the symmetry above and the condition (1.12) with the estimate

$$
\lim _{T \rightarrow+0} \sup _{0<t \leq T}\left\|\langle\cdot\rangle^{a} u(\cdot, t)\right\|_{p, \rho}<\omega\left(\varepsilon_{0}\right) .
$$

Furthermore, for every $\varepsilon^{\prime} \in\left(0, \varepsilon_{0}\right)$, the mapping $\left(\langle\cdot\rangle^{a} u_{0}(\cdot),\langle\cdot\rangle^{2 a} G[F](\cdot, t)\right) \mapsto\langle\cdot\rangle^{a} u(\cdot, t)$ is uniformly continuous from the closed ball in $X$ with center 0 and radius $\varepsilon^{\prime}$ to $B C\left((0, \infty),\left(L^{p, \rho}\left(\mathbb{R}^{2}\right)\right)^{2}\right)$.

Observe that, if the condition $\langle\cdot\rangle^{a} u_{0}(\cdot) \in\left(L^{p, \infty}\left(\mathbb{R}^{2}\right)\right)^{2} \backslash\left(L^{p, \infty-}\left(\mathbb{R}^{2}\right)\right)^{2}$ holds, then the convergence $\langle\cdot\rangle^{a} u(\cdot, t) \rightarrow\langle\cdot\rangle^{a} u_{0}(\cdot)$ in $\left(L^{p, \infty}\left(\mathbb{R}^{2}\right)\right)^{2}$ as $t \rightarrow+0$ does not hold in general, since the space of test functions is not dense in the space $\left\{u(x) \mid\langle x\rangle^{a} u(x) \in\left(L^{p, \infty}\left(\mathbb{R}^{2}\right)\right)^{2}\right\}$. 
In order to treat more general initial data, we rewrite the equation $(1.4)$ in $(0, \infty)$ further. Let $v(x, t) \in B C\left((0, \infty), L_{\sigma}^{2, \infty}\left(\mathbb{R}^{2}\right)\right)$ be the solution of (1.4) satisfying

$$
v(x, t) \rightarrow v_{0} \in L_{\sigma}^{2, \infty}\left(\mathbb{R}^{2}\right) \text { as } t \rightarrow+0 \text { in the sense of distributions, }
$$

and put $w(x, t)=u(x, t)-v(x, t)$. Then we have

$$
\begin{aligned}
\frac{\partial w}{\partial t}(x, t)-\Delta w(x, t)= & P\left(\begin{array}{c}
\frac{\partial H_{1}[0, v+w]}{\partial x_{1}}(x, t)+\frac{\partial H_{2}[0, v+w]}{\partial x_{2}}(x, t) \\
-\frac{\partial H_{1}[0, v+w]}{\partial x_{2}}(x, t)+\frac{\partial H_{2}[0, v+w]}{\partial x_{1}}(x, t)
\end{array}\right) \\
& -P\left(\begin{array}{c}
\frac{\partial H_{1}[0, v]}{\partial x_{1}}(x, t)+\frac{\partial H_{2}[0, v]}{\partial x_{2}}(x, t) \\
-\frac{\partial H_{1}[0, v]}{\partial x_{2}}(x, t)+\frac{\partial H_{2}[0, v]}{\partial x_{1}}(x, t)
\end{array}\right) .
\end{aligned}
$$

For this equation we have the following theorem. In this theorem the symmetry is unnecessary.

Theorem 1.4 For $p$, a and $\rho$ the same as in Theorem 1.1, there exists a positive number $K$ such that the following assertion holds. Let $v(x, t)$ be as above satisfying $\sup _{t>0}\|v(\cdot, t)\|_{2, \infty}<K$.

Then there exists a positive number $\delta_{0}$ and a strictly monotone-increasing function $\psi(\delta)$ on $\left[0, \delta_{0}\right]$ such that $\psi(0)=0$ and the following assertion holds. Suppose that $w_{0}(x) \in L_{\sigma}^{2, \infty}\left(\mathbb{R}^{2}\right)$ satisfies $\left\|w_{0}\right\|_{2, \infty}=\delta<\delta_{0}$. Then there exists a solution $w(x, t)$ of $(1.17)$ in $(0, \infty)$ satisfying

$$
w(x, t) \rightarrow w_{0}(x) \text { as } t \rightarrow+0 \text { in the sense of distributions }
$$

and

$$
\sup _{t>0}\|w(\cdot, t)\|_{2, \infty}+\sup _{t>0} t^{1 / 2-1 / p}\|w(\cdot, t)\|_{p, \infty} \leq \psi(\delta) .
$$

This solution is the unique solution of (1.17)-(1.18) in $(0, \infty)$ with the estimate

$$
\lim _{T \rightarrow+0}\left(\sup _{0<t \leq T}\|w(\cdot, t)\|_{2, \infty}+\sup _{0<t \leq T} t^{1 / 2-1 / p}\|w(\cdot, t)\|_{p, \infty}\right)<\psi\left(\delta_{0}\right) .
$$

Furthermore, for every $\delta^{\prime} \in\left(0, \delta_{0}\right)$, the mapping $w_{0}(x) \mapsto\left(w(\cdot, t), t^{1 / 2-1 / p} w(\cdot, t)\right)$ is uniformly continuous from the closed ball in $L_{\sigma}^{2, \infty}\left(\mathbb{R}^{2}\right)$ with center 0 and radius $\delta^{\prime}$ to

$$
B C\left((0, \infty),\left(L^{2, \infty}\left(\mathbb{R}^{2}\right)\right)^{2}\right) \times B C\left((0, \infty),\left(L^{p, \infty}\left(\mathbb{R}^{2}\right)\right)^{2}\right) .
$$

\section{Reduction to the integral equation}

In order to prove the main results, we rewrite (1.4) into an integral equation. Put

$$
\begin{aligned}
\exp (t \Delta) u_{0}(x) & =\frac{1}{4 \pi t} \int_{\mathbb{R}^{2}} \exp \left(-\frac{(x-y)^{2}}{4 t}\right) u_{0}(y) d y, \\
I[G](x, s, t) & =\exp (s \Delta) P\left(\begin{array}{l}
+\frac{\partial G_{1}}{\partial x_{1}}(\cdot, t-s)+\frac{\partial G_{2}}{\partial x_{2}}(\cdot, t-s)-\frac{\partial G_{3}}{\partial x_{2}}(\cdot, t-s) \\
-\frac{\partial G_{1}}{\partial x_{2}}(\cdot, t-s)+\frac{\partial G_{2}}{\partial x_{1}}(\cdot, t-s)+\frac{\partial G_{3}}{\partial x_{1}}(\cdot, t-s)
\end{array}\right), \\
I_{0}[G](x, t, T) & =\int_{0}^{T} I[G](x, s, t) d s, I_{1}[G](x, t)=\lim _{T \rightarrow \infty} I_{0}[G](x, t, T) .
\end{aligned}
$$


Then we can formally rewrite $(1.4)$ in $(-\infty, \infty)$ into the form

$$
u(x, t)=I_{1}[H[F, u]](x, t) \text { for } t \in \mathbb{R}
$$

and (1.4) in $(0, \infty)$ with $(0.2)$ into

$$
u(x, t)=\exp (t \Delta) u_{0}+I_{0}[H[F, u]](x, t, t) \text { for } t>0
$$

respectively.

We first introduce a proposition concerning the estimate of heat kernel on weighted $L^{p}$ spaces.

Proposition 2.1 Suppose that $1<p<\infty$ and that either $1 \leq q<\infty, q=\infty$ - or $q=\infty$. If a satisfies $-n / p<a<n(1-1 / p)$, then we have the following assertions:

(i) There exists a positive constant $C_{a, p}$ such that the estimate $\left\|\langle\cdot\rangle^{a} \exp (t \Delta) u\right\|_{p, q} \leq$ $C_{a, p}\left\|\langle\cdot\rangle^{a} u\right\|_{p, q}$ holds for every $t \geq 0$ and every $u(x)$ such that $\langle\cdot\rangle^{a} a \in L^{p, q}\left(\mathbb{R}^{2}\right)$.

(ii) If either $1 \leq q<\infty$ or $q=\infty$-, then we have $\langle\cdot\rangle^{a} \exp (t \Delta) u \rightarrow\langle\cdot\rangle^{a} u$ in $L^{p, q}\left(\mathbb{R}^{2}\right)$ as $t \rightarrow+0$.

Proof We first show Assertion (i) for $q=p$. In this case $-n<a p<n(p-1)$, and hence $\langle\cdot\rangle^{a p}$ is an $A_{p}$ weight. Then weighted boundedness of the Fourier multiplier implies the estimate

$$
\begin{aligned}
\left\|\langle\cdot\rangle^{a} \exp (t \Delta) u\right\|_{p} & =\left\|\mathscr{F}^{-1}\left[\exp \left(-t|\xi|^{2}\right) \mathscr{F}[u](\xi)\right]\right\|_{L^{p}\left(\langle\cdot)^{a p}\right)} \\
& \leq C_{a, p}^{\prime}\|u\|_{L^{p}\left(\langle\cdot)^{a p}\right)}=C_{a, p}^{\prime}\left\|\langle\cdot\rangle^{a} u\right\|_{p},
\end{aligned}
$$

from which the conclusion for $q=p$ follows at once.

We next consider the general case of Assertion (i). Choose $p_{0}, p_{1}$ such that $p_{0}<p<p_{1}$ and that $-n / p_{j}<a<n\left(1-1 / p_{j}\right)$ for $j=0,1$. Then Assertion (i) for $q_{j}=p_{j}$ yields the estimate

$$
\left\|\langle\cdot\rangle^{a} \exp (t \Delta) u\right\|_{p_{j}} \leq C_{a, p_{j}}^{\prime}\left\|\langle\cdot\rangle^{a} u\right\|_{p_{j}}
$$

for $j=0,1$. This fact implies that the mapping $v \mapsto\langle\cdot\rangle^{a} \exp (t \Delta)\left(\langle\cdot\rangle^{-a} v\right)$ is bounded from $L^{p_{j}}\left(\mathbb{R}^{2}\right)$ into itself with norm independent of $t \geq 0$. Hence, by real interpolation, we see that the mapping above is bounded from $L^{p, q}\left(\mathbb{R}^{2}\right)$ into itself with norm independent of $t \geq 0$. This fact implies $\left\|\langle\cdot\rangle^{a} \exp (t \Delta) u\right\|_{p, q} \leq C_{a, p}\left\|\langle\cdot\rangle^{a} u\right\|_{p, q}$ with a positive constant $C_{a, p}$ independent of $q$. This yields the Assertion (i) for general case.

We turn to the Assertion (ii). From the assumption we see that, for every fixed $\varepsilon>0$, there exists a function $\varphi \in C_{0}^{\infty}\left(\mathbb{R}^{2}\right)$ such that $\left\|\langle\cdot\rangle^{a} u-\varphi\right\|_{p, q}<\varepsilon / 2\left(C_{a, p}+1\right)$. Then we have

$$
\left\|\langle\cdot\rangle^{a}(\exp (t \Delta) u-u)\right\|_{p, q} \leq I_{1}+I_{2}+I_{3}
$$

where

$$
\begin{aligned}
& I_{1}=\left\|\langle\cdot\rangle^{a} \exp (t \Delta)\left(u-\langle\cdot\rangle^{-a} \varphi\right)\right\|_{p, q}, \\
& I_{2}=\left\|\langle\cdot\rangle^{a}(\exp (t \Delta)-1)\langle\cdot\rangle^{-a} \varphi\right\|_{p, q}
\end{aligned}
$$

and

$$
I_{3}=\left\|\varphi-\langle\cdot\rangle^{a} u\right\|_{p, q} .
$$


Then Assertion (i) implies $I_{1} \leq C_{a, p}\left\|\langle\cdot\rangle^{a} u-\varphi\right\|_{p, q}$. It follows that

$$
I_{1}+I_{3}<\frac{\varepsilon}{2}
$$

On the other hand, we have

$$
\begin{aligned}
I_{2} & =\left\|\langle\cdot\rangle^{a} \int_{0}^{t} \frac{d}{d \tau} \exp (\tau \Delta)\langle\cdot\rangle^{-a} \varphi d \tau\right\|_{p, q} \\
& \leq t \sup _{\tau \in[0, t]}\left\|\langle\cdot\rangle^{a} \exp (\tau \Delta) \Delta\langle\cdot\rangle^{-a} \varphi\right\|_{p, q} \\
& \leq C_{p, q} t\left\|\langle\cdot\rangle^{a} \Delta\langle\cdot\rangle^{-a} \varphi\right\|_{p, q} .
\end{aligned}
$$

We now put $T=\varepsilon / 2 C_{p, q}\left\|\langle\cdot\rangle^{a} \Delta\langle\cdot\rangle^{-a} \varphi\right\|_{p, q}$. Then we have $I_{2} \leq \varepsilon / 2$ for every $t \in[0, T]$. This estimate, together with (2.3) and (2.4), yields $\|\langle\cdot\rangle(\exp (t \Delta) u-u)\|_{p, q}<\varepsilon$ provided $t \in[0, T]$. Since $\varepsilon>0$ is arbitrary, this completes the proof of Assertion (ii).

Then the following proposition, which will be proved at the end of this section, justifies the transformation above rigorously. Although some of the assumptions are unnecessary in the proof, the statement is designed so that it is directly applicable in later sections

Proposition 2.2 Suppose that $1<q<2 \leq r<\infty$ and that $1 \leq \rho \leq \infty$. Then we have the following assertions:

(i) Suppose that $s \in \mathbb{R},\langle\cdot\rangle^{a} u_{0}(\cdot) \in\left(L^{2, \rho}\left(\mathbb{R}^{2}\right)+L^{r, \rho}\left(\mathbb{R}^{2}\right)\right)^{2}$, and

$$
\langle\cdot\rangle^{2 a} \Phi(\cdot, t) \in B C\left((s, \infty),\left(L^{q, \rho}\left(\mathbb{R}^{2}\right)\right)^{2}\right) .
$$

Then the function $u(x, t)$ defined by the formula

$$
u(\cdot, t)=\exp ((t-s) \Delta) u_{0}+\int_{0}^{t-s} \exp (\tau \Delta) \Phi(\cdot, t-\tau) d \tau
$$

satisfies $\langle\cdot\rangle^{a} u(\cdot, t) \in C\left((s, \infty),\left(L^{r, \rho}\left(\mathbb{R}^{2}\right)\right)^{2}\right)$, and

$$
\begin{aligned}
\frac{\partial u}{\partial t}(x, t)-\Delta u(x, t) & =\Phi(x, t) \text { in } \mathbb{R}^{2} \times(s, \infty), \\
u(x, t) & \rightarrow u_{0}(x) \text { as } t \rightarrow s+0
\end{aligned}
$$

in the sense of distributions. Moreover, if $u_{0} \in\left(L^{r, \tilde{\rho}}\left(\mathbb{R}^{2}\right)\right)^{2}$ as well, where $\tilde{\rho}$ is given by (1.14), then we have

$$
u(\cdot, t) \rightarrow u_{0} \text { in }\left(L^{r, \rho}\left(\mathbb{R}^{2}\right)\right)^{2} \text { as } t \rightarrow s+0 .
$$

Conversely, if

$$
u(\cdot, t) \in C\left((s, \infty),\left(L^{r, \rho}\left(\mathbb{R}^{2}\right)\right)^{2}\right)
$$

is a solution of (2.6)-(2.7), then $u(x, t)$ is given by (2.5).

(ii) Suppose that $\Phi(\cdot, t) \in B C\left(\mathbb{R},\left(L^{q, \rho}\left(\mathbb{R}^{2}\right)\right)^{2}\right)$. and that, for every $t \in \mathbb{R}$, the limit

$$
\lim _{T \rightarrow \infty} \int_{0}^{T} \exp (\tau \Delta) \Phi(\cdot, t-\tau) d \tau
$$


exists in the weak-* topology of $\left(L^{r, \rho}\left(\mathbb{R}^{2}\right)\right)^{2}$. Then the limit $u(\cdot, t)$ belongs to $B C\left(\mathbb{R},\left(L^{r, \rho}\left(\mathbb{R}^{2}\right)\right)^{2}\right)$, and is a solution of the problem

$$
\frac{\partial u}{\partial t}(x, t)-\Delta u(x, t)=\Phi(x, t) \text { in } \mathbb{R}^{2} \times \mathbb{R}
$$

in the sense of distributions. Conversely, if

$$
u(\cdot, t) \in B C\left(\mathbb{R},\left(L^{r, \rho}\left(\mathbb{R}^{2}\right)\right)^{2}\right)
$$

is a solution of (2.10), then (2.9) exists in the weak-* topology for every $t \in \mathbb{R}$, and the limit coincides with $u(\cdot, t)$.

In order to solve (2.1) and (2.2), consider the mappings

$$
T_{1}: u(x, t) \mapsto I_{1}[H[F, u]](x, t)
$$

and

$$
T_{2}: u(x, t) \mapsto \exp (t \Delta) u_{0}(x, t)+I_{0}[H[F, u]](x, t, t)
$$

respectively, and assume the conditions (0.5) and (0.6), and $u(x, t)$ satisfies $(\mathrm{C} 4 \mathrm{E})$. Then we have

$$
\begin{aligned}
& H_{1}[0, u]\left(x^{\perp}, t\right)=\frac{u_{1}\left(x^{\perp}, t\right)^{2}-u_{2}\left(x^{\perp}, t\right)^{2}}{2}=\frac{u_{2}(x, t)^{2}-u_{1}(x, t)^{2}}{2}=-H_{1}[0, u](x, t), \\
& H_{2}[0, u]\left(x^{\perp}, t\right)=u_{1}\left(x^{\perp}, t\right) u_{2}\left(x^{\perp}, t\right)=-u_{2}(x, t) u_{1}(x, t)=-H_{2}[0, u](x, t) .
\end{aligned}
$$

It follows that $H_{1}[F, u](x, t)$ and $H_{2}[F, u](x, t)$ satisfy $(0.5)$.

If $b \geq 0$, the mapping $f(x) \mapsto\langle x\rangle^{-b} f(x)$ is bounded on $L^{q, \rho}\left(\mathbb{R}^{2}\right)$ onto itself. Hence the condition

$$
\begin{array}{r}
\langle\cdot\rangle^{a} u(\cdot, t) \in B C\left(I,\left(L^{p, \rho}\left(\mathbb{R}^{2}\right)\right)^{2}\right), \\
\langle\cdot\rangle^{2 a} G[F](\cdot, t) \in B C\left(I,\left(L^{p / 2, \rho}\left(\mathbb{R}^{2}\right)\right)^{3}\right)
\end{array}
$$

for $I=\mathbb{R}$, together with the existence of the limit

$$
\lim _{T \rightarrow \infty} \int_{0}^{T} \exp (\tau \Delta)\langle\cdot\rangle^{2 a} G[F](\cdot, t-\tau) d \tau
$$

with respect to the weak-* topology of $\left(L^{p / 2, \rho}\left(\mathbb{R}^{2}\right)\right)^{2}$ and the continuous dependence of the limit in $\left(L^{p / 2, \rho}\left(\mathbb{R}^{2}\right)\right)^{2}$ with respect to $t$, implies the assumptions of Proposition 2.2, (ii), and (2.11) for $I=(0, \infty)$ implies that of Proposition 2.2, (i). Hence Theorems 1.1 and 1.3 reduce to the following theorems respectively.

Theorem 2.3 Let p, a and $\rho$ be the same as in Theorem 1.1. Then there exist a positive constant $\varepsilon_{0}$ and a strictly monotone-increasing function $\omega(s)$ on $\left[0, \varepsilon_{0}\right]$ such that $\omega(0)=0$ and that the following assertion holds. Suppose that the function $G[F](x, t)=$ $\left(G_{1}[F](x, t), G_{2}[F](x, t), G_{3}[F](x, t)\right)$ satisfies $\langle\cdot\rangle^{2 a} G[F](\cdot, t) \in B C\left(\mathbb{R},\left(L^{p / 2, \rho}\left(\mathbb{R}^{2}\right)\right)^{3}\right)$ with the estimate (1.6). We moreover assume that $G_{1}[F](\cdot, t), G_{2}[F](\cdot, t)$ satisfy $(0.5)$ and that $G_{3}[F](\cdot, t)$ satisfies $(0.6)$ for every $t \in \mathbb{R}$. 
Then there exists a solution $u(\cdot, t)$ of (2.1) such that the symmetry condition $(\mathrm{C} 4 \mathrm{E})$ holds for every $t \in \mathbb{R}$ and that (1.7) with the estimate (1.8). This solution is the unique solution of (2.1) satisfying the symmetry above and the condition (1.7) with the estimate (1.9).

Furthermore, for every $\varepsilon^{\prime} \in\left(0, \varepsilon_{0}\right)$, the mapping $\langle\cdot\rangle^{2 a} G[F](\cdot, t) \mapsto\langle\cdot\rangle^{a} u(\cdot, t)$ is uniformly continuous from the closed ball in $B C\left(\mathbb{R},\left(L^{p / 2, \rho}\left(\mathbb{R}^{2}\right)\right)^{3}\right)$ with center 0 and radius $\varepsilon^{\prime}$ to $B C\left(\mathbb{R},\left(L^{p, \rho}\left(\mathbb{R}^{2}\right)\right)^{2}\right)$.

Theorem 2.4 Let $p, a$ and $\rho$ be the same as in Theorem 2.3. Suppose that $u_{0}(x)$ satisfies $(\mathrm{C} 4 \mathrm{E})$ and $\nabla \cdot u_{0}(x)=0$, and that $G_{1}[F](\cdot, t), G_{2}[F](\cdot, t)$ satisfy $(0.5)$ and that $G_{3}[F](\cdot, t)$ satisfies (0.6) for every $t>0$. Furthermore, putting

$$
G[F](x, t)=\left(G_{1}[F](x, t), G_{2}[F](x, t), G_{3}[F](x, t)\right),
$$

we assume that $\left(\langle\cdot\rangle^{a} u_{0}(\cdot),\langle\cdot\rangle^{2 a} G[F](\cdot, t)\right) \in X$ with the estimate $(1.10)$.

Then there exists a solution $u(\cdot, t)$ of $(2.2)$ such that the symmetry condition $(\mathrm{C} 4 \mathrm{E})$ holds for every $t>0$ and that (1.12) with the estimate (1.13) holds. This solution is the unique solution of (2.2) with satisfying the symmetry above and the condition (1.12) with the estimate (1.15). Furthermore, for every $\varepsilon^{\prime} \in\left(0, \varepsilon_{0}\right)$, the mapping

$$
\left(\langle\cdot\rangle^{a} u_{0}(\cdot),\langle\cdot\rangle^{2 a} G[F](\cdot, t)\right) \mapsto\langle\cdot\rangle^{a} u(\cdot, t)
$$

is uniformly continuous from the closed ball in $X$ with center $O$ and radius $\varepsilon^{\prime}$ to $B C\left((0, \infty),\left(L^{p, \rho}\left(\mathbb{R}^{2}\right)\right)^{2}\right)$.

In the same way as in (2.2), we can formally rewrite (1.17) into

$$
w(x, t)=\exp (t \Delta) w_{0}+I_{0}[H[0, v+w]](x, t, t)-I_{0}[H[0, v]](x, t, t) \text { for } t>0 .
$$

Moreover, if we put $r=p$ and $q=2 p /(2+p)$, we see that

$$
v(\cdot, t), w(\cdot, t) \in B C\left((0, \infty), L_{\sigma}^{p, \rho}\left(\mathbb{R}^{2}\right) \cap L_{\sigma}^{2, \infty}\left(\mathbb{R}^{2}\right)\right)
$$

implies the assumptions of Proposition 2.2, (i). Hence Theorem 1.4 reduces to the following theorem.

Theorem 2.5 Let $p$, $a$ and $\rho$ be the same as in Theorem 1.1, and let $K$ and $v(x, t)$ be the same as in Theorem 1.4. Then there exists a positive number $\delta_{0}$ and a strictly monotone-increasing function $\psi(\delta)$ on $\left[0, \delta_{0}\right]$ such that $\psi(0)=0$ and the following assertion holds. Suppose that $w_{0}(x) \in L_{\sigma}^{2, \infty}\left(\mathbb{R}^{2}\right)$ satisfies $\left\|w_{0}\right\|_{2, \infty}=\delta<\delta_{0}$. Then there exists a solution $w(x, t)$ of (2.12) satisfying

$$
\sup _{t>0}\|w(\cdot, t)\|_{2, \infty}+\sup _{t>0} t^{1 / 2-1 / p}\|w(\cdot, t)\|_{p, \infty} \leq \psi(\delta) .
$$

This solution is the unique solution of (2.12) satisfying (1.19).

Furthermore, for every $\delta^{\prime} \in\left(0, \delta_{0}\right)$, the mapping $w_{0}(x) \mapsto\left(u(\cdot, t), t^{1 / 2-1 / p} u(\cdot, t)\right)$ is uniformly continuous from the closed ball in $L_{\sigma}^{2, \infty}\left(\mathbb{R}^{2}\right)$ with center 0 and radius $\delta^{\prime}$ to

$$
B C\left((0, \infty),\left(L^{2, \infty}\left(\mathbb{R}^{2}\right)\right)^{2}\right) \times B C\left((0, \infty),\left(L^{p, \infty}\left(\mathbb{R}^{2}\right)\right)^{2}\right) .
$$


Proof of Proposition 2.2 We first prove Assertion (i). Suppose that $u(x, t)$ is a solution of (2.5) in $(s, \infty)$. We first prove the continuity. Fix $t_{1}>s$ arbitrarily. Then we have

$$
\exp \left(\left(t_{1}-s\right) \Delta\right) u_{0}, \Delta \exp \left(\left(t_{1}-s\right) \Delta\right) u_{0} \in\left(L^{r, \rho}\left(\mathbb{R}^{2}\right)\right)^{2}
$$

It follows that $\exp \left(\left(t_{1}-s\right) \Delta\right) u_{0}$ belongs to the closure of $D(\Delta)$ as a closable operator in $\left(L^{p, \rho}\left(\mathbb{R}^{2}\right)\right)^{2}$ even in the case $\rho=\infty$. Hence $\exp ((t-s) \Delta) u_{0}$ is bounded and continuous in $\left(L^{r, \rho}\left(\mathbb{R}^{2}\right)\right)^{2}$ on $\left[t_{1}, \infty\right)$. Since $t_{1}>s$ is arbitrary, it follows that $\exp ((t-s) \Delta) u_{0}$ is continuous on $(s, \infty)$. If $u_{0}(x) \in\left(L^{r, \tilde{\rho}}\left(\mathbb{R}^{2}\right)\right)^{2}$ as well, where $\tilde{\rho}$ is given in (1.14), then $u_{0}(x)$ is in the closure of $D(\Delta)$ in $\left(L^{r, \rho}\left(\mathbb{R}^{2}\right)\right)^{2}$, and hence

$$
\exp ((t-s) \Delta) u_{0} \rightarrow u_{0} \text { in }\left(L^{r, \rho}\left(\mathbb{R}^{2}\right)\right)^{2} \text { as } t \rightarrow s+0
$$

Next, from the estimate

$$
\begin{aligned}
\left\|\int_{0}^{t-s} \exp (\tau \Delta) \Phi(\cdot, t-\tau) d \tau\right\|_{r, \rho} & \leq \int_{0}^{t-s}\|\exp (\tau \Delta) \Phi(\cdot, t-\tau)\|_{r, \rho} d \tau \\
& \leq C \int_{0}^{t-s} \tau^{-1 / q+1 / r}\|\Phi(\cdot, t-\tau)\|_{q, \rho} d s \\
& \leq C(t-s)^{1-1 / q+1 / r} \sup _{\tau>s}\|\Phi(\cdot, \tau)\|_{q, \rho}
\end{aligned}
$$

we see that

$$
\int_{0}^{t-s} \exp (\tau \Delta) \Phi(\cdot, t-\tau) d \tau \rightarrow 0 \text { in }\left(L^{r, \rho}\left(\mathbb{R}^{2}\right)\right)^{2} \text { as } t \rightarrow s+0 .
$$

Next we prove the continuity of the term $\int_{0}^{t-s} \exp (\tau \Delta) \Phi(\cdot, t-\tau) d \tau$ at $t=t_{0}>s$. To this end we fix $\delta \in\left(0,\left(t_{0}-s\right) / 2\right)$, and suppose that $t_{0}-\delta<t_{1} \leq t_{0} \leq t_{2}<t_{0}+\delta$ and that $t_{1}<t_{2}$. Then we have

$$
\begin{aligned}
I\left(t_{1}, t_{2}\right)= & \int_{0}^{t_{2}-s} \exp (\tau \Delta) \Phi\left(\cdot, t_{2}-\tau\right) d \tau \\
& -\int_{0}^{t_{1}-s} \exp (\tau \Delta) \Phi\left(\cdot, t_{1}-\tau\right) d \tau=I_{1}+I_{2}+I_{3},
\end{aligned}
$$

where

$$
\begin{aligned}
& I_{1}=\int_{0}^{t_{1}-s-\delta} \exp (\tau \Delta)\left(\Phi\left(\cdot, t_{2}-\tau\right)-\Phi\left(\cdot, t_{1}-\tau\right)\right) d \tau \\
& I_{2}=\int_{t_{1}-s-\delta}^{t_{2}-s} \exp (\tau \Delta) \Phi\left(\cdot, t_{2}-\tau\right) d \tau \\
& I_{3}=-\int_{t_{1}-s-\delta}^{t_{1}-s} \exp (\tau \Delta) \Phi\left(\cdot, t_{1}-\tau\right) d \tau .
\end{aligned}
$$


Then we have

$$
\begin{aligned}
\left\|I_{3}\right\|_{r, \rho} & \leq \int_{t_{1}-s-\delta}^{t_{2}-s}\left\|\exp (\tau \Delta) \Phi\left(\cdot, t_{1}-\tau\right)\right\|_{r, \rho} d \tau \\
& \leq \int_{t_{1}-s-\delta}^{t_{1}-s+2 \delta} C \tau^{-1 / q+1 / r}\left\|\Phi\left(\cdot, t_{1}-\tau\right)\right\|_{q, \rho} d \tau \\
& \leq C \delta^{1-1 / q+1 / r} \sup _{t>s}\|\Phi(\cdot, t)\|_{q, \rho} .
\end{aligned}
$$

Next, since $t_{2}-\left(t_{1}-\delta\right)<3 \delta$, we have

$$
\left\|I_{2}\right\|_{r, \rho} \leq C \delta^{1-1 / q+1 / r} \sup _{t>s}\|\Phi(\cdot, t)\|_{q, \rho}
$$

in the same way. Hence these two terms can be taken arbitrarily small if we take $\delta>0$ sufficiently small. Next we have

$$
\begin{aligned}
I_{1} & \leq \int_{0}^{t_{1}-s-\delta}\left\|\exp (\tau \Delta)\left(\Phi\left(\cdot, t_{2}-\tau\right)-\Phi\left(\cdot, t_{1}-\tau\right)\right)\right\|_{r, \rho} d \tau \\
& \leq \int_{0}^{t_{1}-s-\delta} \tau^{-1 / q+1 / r}\left\|\Phi\left(\cdot, t_{2}-\tau\right)-\Phi\left(\cdot, t_{1}-\tau\right)\right\|_{q, \rho} d \tau \\
& \leq \int_{0}^{t_{0}-s} \tau^{-1 / q+1 / r} d \tau \sup _{s+\delta \leq \tau \leq t_{0}}\left\|\Phi\left(\cdot, \tau+t_{2}-t_{1}\right)-\Phi(\cdot, \tau)\right\|_{q, \rho} \\
& =\left(t_{0}-s\right)^{1-1 / q+1 / r} \sup _{s+\delta \leq \tau \leq t_{0}}\left\|\Phi\left(\cdot, \tau+t_{2}-t_{1}\right)-\Phi(\cdot, \tau)\right\|_{q, \rho}
\end{aligned}
$$

Once $t_{0}$ and $\delta$ are fixed, the term $\left\|I_{1}\right\|_{r, \rho}$ can be taken arbitrarily small if $t_{2}-t_{1} \rightarrow$ +0 , since $\Phi(\cdot, t)$ is uniformly continuous in $\left(L^{q, \rho}\left(\mathbb{R}^{2}\right)\right)^{2}$ on $\left[s+\delta, t_{0}+\delta\right]$. Hence $\int_{0}^{t-s} \exp (\tau \Delta) \Phi(\cdot, t-\tau) d \tau$ is continuous at $t=t_{0}>s$. This completes the proof of the required continuity.

We next show that $u(x, t)$ satisfies (2.6)-(2.7). Fix $t_{0}, t_{1}$ such that $s<t_{1}<t_{0}$, and assume that $t_{1}<t_{2} \leq t_{0} \leq t_{3}<\infty$ and $t_{2}<t_{3}$. Then, for every $\varphi(x) \in\left(C_{0}^{\infty}\left(\mathbb{R}^{2}\right)\right)^{2}$, we have

$$
\left(\frac{u\left(\cdot, t_{3}\right)-u\left(\cdot, t_{2}\right)}{t_{3}-t_{2}}, \varphi\right)=J_{1}+J_{2}+J_{3},
$$

where

$$
\begin{aligned}
& J_{1}=\left(\frac{\left\{\exp \left(\left(t_{3}-t_{2}\right) \Delta\right)-1\right\} \exp \left(\left(t_{2}-s\right) \Delta\right) u_{0}}{t_{3}-t_{2}}, \varphi\right) \\
& J_{2}=\frac{1}{t_{3}-t_{2}}\left(\int_{t_{3}-t_{2}}^{t_{3}-s} \exp (\tau \Delta) \Phi\left(\cdot, t_{3}-\tau\right) d \tau-\int_{0}^{t_{2}-s} \exp (\tau \Delta) \Phi\left(\cdot, t_{2}-\tau\right) d \tau, \varphi\right)
\end{aligned}
$$

and

$$
J_{3}=\frac{1}{t_{3}-t_{2}}\left(\int_{0}^{t_{3}-t_{2}} \exp (\tau \Delta) \Phi\left(\cdot, t_{3}-\tau\right) d \tau, \varphi\right)
$$

In the sequel the limits are taken either $t_{2}=t_{0}, t_{3} \rightarrow t_{0}+0$ or $t_{2} \rightarrow t_{0}-0$ and $t_{3}=t_{0}$. We first consider $J_{1}$. We can write

$$
J_{1}=\left(\exp \left(\left(t_{2}-t_{1}\right) \Delta\right) \exp \left(\left(t_{1}-s\right) \Delta\right) u_{0}, \frac{\left\{\exp \left(\left(t_{3}-t_{2}\right) \Delta\right)-1\right\} \varphi}{t_{3}-t_{2}}\right)
$$


Since $\exp \left(\left(t_{1}-s\right) \Delta\right) u_{0} \in\left(L^{r, \rho}\left(\mathbb{R}^{2}\right)\right)^{2}$, we have

$$
\begin{gathered}
\exp \left(\left(t_{2}-t_{1}\right) \Delta\right) \exp \left(\left(t_{1}-s\right) \Delta\right) u_{0} \rightarrow \\
\exp \left(\left(t_{0}-s\right) \Delta\right) u_{0} * \text {-weakly in }\left(L^{r, \rho}\left(\mathbb{R}^{2}\right)\right)^{2} .
\end{gathered}
$$

On the other hand, we have

$$
\begin{aligned}
\frac{1}{t_{3}-t_{2}}\left\{\exp \left(\left(t_{3}-t_{2}\right) \Delta\right)-1\right\} \varphi & =\frac{1}{t_{3}-t_{2}} \int_{0}^{t_{3}-t_{2}} \Delta \exp (\tau \Delta) \varphi d \tau \\
& =\int_{0}^{1} \exp \left(\theta\left(t_{3}-t_{2}\right) \Delta\right) \Delta \varphi d \theta
\end{aligned}
$$

Since $\varphi \in\left(C_{0}^{\infty}\left(\mathbb{R}^{2}\right)\right)^{2}$, we see that $\exp \left(\theta\left(t_{3}-t_{2}\right) \Delta\right) \Delta \varphi \rightarrow \Delta \varphi$ in $\left(L^{r /(r-1), \rho^{\prime}}\left(\mathbb{R}^{2}\right)\right)^{2}$, where $\rho^{\prime}$ is defined by (1.5). It follows that

$$
\frac{1}{t_{3}-t_{2}}\left\{\exp \left(\left(t_{3}-t_{2}\right) \Delta\right)-1\right\} \varphi \rightarrow \Delta \varphi \text { in }\left(L^{r /(r-1), \rho^{\prime}}\left(\mathbb{R}^{2}\right)\right)^{2}
$$

From (2.16), (2.17) and (2.18) we conclude

$$
J_{1} \rightarrow\left(\exp \left(\left(t_{0}-s\right) \Delta\right) u_{0}, \Delta \varphi\right)
$$

We next consider $J_{2}$. We can write

$$
\begin{aligned}
J_{2} & =\frac{1}{t_{3}-t_{2}}\left(\int_{s}^{t_{2}}\left\{\exp \left(\left(t_{3}-\tau\right) \Delta\right)-\exp \left(\left(t_{2}-\tau\right) \Delta\right)\right\} \Phi(\cdot, \tau) d \tau, \varphi\right) \\
& =\int_{s}^{t_{2}}\left(\Phi(\cdot, \tau), \frac{1}{t_{3}-t_{2}}\left\{\exp \left(\left(t_{3}-\tau\right) \Delta\right)-\exp \left(\left(t_{2}-\tau\right) \Delta\right)\right\} \varphi d \tau\right) .
\end{aligned}
$$

We now split $J_{2}=J_{2,1}+J_{2,2}$, where

$$
J_{2,1}=\int_{s}^{t_{1}}\left(\Phi(\cdot, \tau), \frac{1}{t_{3}-t_{2}}\left\{\exp \left(\left(t_{3}-\tau\right) \Delta\right)-\exp \left(\left(t_{2}-\tau\right) \Delta\right)\right\} \varphi d \tau\right)
$$

and

$$
J_{2,2}=\int_{t_{1}}^{t_{2}}\left(\Phi(\cdot, \tau), \frac{1}{t_{3}-t_{2}}\left\{\exp \left(\left(t_{3}-\tau\right) \Delta\right)-\exp \left(\left(t_{2}-\tau\right) \Delta\right)\right\} \varphi d \tau\right)
$$

Then we have

$$
\begin{aligned}
\frac{1}{t_{3}-t_{2}}\left\{\exp \left(\left(t_{3}-\tau\right) \Delta\right)-\exp \left(\left(t_{2}-\tau\right) \Delta\right)\right\} \varphi & =\frac{1}{t_{3}-t_{2}} \int_{t_{2}-\tau}^{t_{3}-\tau} \Delta \exp (\rho \Delta) \varphi d \rho \\
& =\int_{0}^{1} \exp \left\{\left(\theta\left(t_{3}-t_{2}\right)+\left(t_{2}-\tau\right)\right) \Delta\right\} \Delta \varphi d \theta .
\end{aligned}
$$

Substituting this equality into (2.20) and (2.21) we obtain

$$
J_{2,1}=\int_{s}^{t_{1}}\left(\Phi(\cdot, \tau), \int_{0}^{1} \exp \left\{\left(\theta\left(t_{3}-t_{2}\right)+\left(t_{2}-\tau\right)\right) \Delta\right\} \varphi d \tau\right)
$$

and

$$
J_{2,2}=\int_{t_{1}}^{t_{2}}\left(\Phi(\cdot, \tau), \int_{0}^{1} \exp \left\{\left(\theta\left(t_{3}-t_{2}\right)+\left(t_{2}-\tau\right)\right) \Delta\right\} \varphi d \tau\right)
$$


If $\tau \leq t_{1}$, it follows that

$$
\begin{aligned}
& \exp \left\{\left(\theta\left(t_{3}-t_{2}\right)+\left(t_{2}-\tau\right)\right) \Delta\right\} \Delta \varphi \\
& \quad=\exp \left\{\left(\theta\left(t_{3}-t_{2}\right)+\left(t_{2}-t_{1}\right)\right) \Delta\right\} \exp \left(\left(t_{1}-\tau\right) \Delta\right) \Delta \varphi \\
& \rightarrow \exp \left(\left(t_{0}-t_{1}\right) \Delta\right) \exp \left(\left(t_{1}-\tau\right) \Delta\right) \Delta \varphi=\exp \left(\left(t_{0}-\tau\right) \Delta\right) \Delta \varphi .
\end{aligned}
$$

Substituting this into (2.22) we conclude that

$$
\begin{gathered}
J_{2,1} \rightarrow \int_{s}^{t_{1}}\left(\Phi(\cdot, \tau), \exp \left(\left(t_{0}-\tau\right) \Delta\right) \Delta \varphi\right) d \tau \\
=\left(\int_{t_{0}-t_{1}}^{t_{0}-s} \exp (\tau \Delta) \Phi\left(\cdot, t_{0}-\tau\right) d \tau, \Delta \varphi\right) .
\end{gathered}
$$

Next, (2.23) implies

$$
\begin{aligned}
\left|J_{2,2}\right| & \leq\left(t_{2}-t_{1}\right) \sup _{t>s}\|\Phi(\cdot, t)\|_{q, \rho}\|\Delta \varphi\|_{q /(q-1), \rho^{\prime}} \\
& \leq\left(t_{0}-t_{1}\right) \sup _{t>s}\|\Phi(\cdot, t)\|_{q, \rho}\|\Delta \varphi\|_{q /(q-1), \rho^{\prime}} .
\end{aligned}
$$

On the other hand, we have

$$
\begin{aligned}
& \left|\left(\int_{0}^{t_{0}-t_{1}} \exp (\tau \Delta) \Phi\left(\cdot, t_{0}-\tau\right) d \tau, \Delta \varphi\right)\right| \\
& \quad \leq\left(t_{1}-t_{0}\right) \sup _{t>s}\|\Phi(\cdot, t)\|_{q, \rho}\|\Delta \varphi\|_{q /(q-1), \rho^{\prime}}
\end{aligned}
$$

It follows from (2.19), (2.24), (2.25) and (2.26) that

$$
\begin{aligned}
& \lim \sup \left|J_{1}+J_{2}-\left(u\left(\cdot, t_{0}\right), \Delta \varphi\right)\right| \\
& =\lim \sup \mid J_{1}+J_{2,1}+J_{2,2} \\
& \quad-\left(\exp \left(\left(t_{0}-s\right) \Delta\right) u_{0}+\int_{0}^{t_{0}-s} \exp (\tau \Delta) \Phi\left(\cdot, t_{0}-\tau\right) d \tau, \Delta \varphi\right) \mid \\
& \quad \leq 2\left(t_{1}-t_{0}\right) \sup _{t>s}\|\Phi(\cdot, t)\|_{q, \rho}\|\Delta \varphi\|_{q /(q-1), \rho^{\prime}},
\end{aligned}
$$

We finally rewrite $J_{3}=J_{3,1}+J_{3,2}$, where

$$
J_{3,1}=\frac{1}{t_{3}-t_{2}}\left(\int_{0}^{t_{3}-t_{2}}(\exp (\tau \Delta)-1) \Phi\left(\cdot, t_{3}-\tau\right), \varphi\right)
$$

and

$$
J_{3,2}=\frac{1}{t_{3}-t_{2}}\left(\int_{0}^{t_{3}-t_{2}} \Phi\left(\cdot, t_{3}-\tau\right), \varphi\right) .
$$

Then we can estimate

$$
\begin{aligned}
\left|J_{3,1}\right| & \leq \frac{1}{t_{3}-t_{2}} \int_{0}^{t_{3}-t_{2}}\left\|\Phi\left(\cdot, t_{3}-\tau\right)\right\|_{q, \rho} \sup _{0 \leq \tau \leq t_{3}-t_{2}}\|(\exp (\tau \Delta)-1) \varphi\|_{q /(q-1), \rho^{\prime}} d \tau \\
& \leq \sup _{t>s}\|\Phi(\cdot, t)\|_{q, \rho} \sup _{0 \leq \tau \leq t_{3}-t_{2}}\|(\exp (\tau \Delta)-1) \varphi\|_{q /(q-1), \rho^{\prime}} \rightarrow 0 .
\end{aligned}
$$


Finally, since $F(\cdot, t)$ is continuous at $t=t_{0}$ in $\left(L^{q, \rho}\left(\mathbb{R}^{2}\right)\right)^{2}$, we conclude that $J_{3,2} \rightarrow$ $\left(\Phi\left(\cdot, t_{0}\right), \varphi\right)$. This implies $J_{3} \rightarrow\left(\Phi\left(\cdot, t_{0}\right), \varphi\right)$. Substituting this fact and (2.27) into (2.15), we obtain

$$
\begin{aligned}
& \lim \sup \left|\left(\frac{u\left(\cdot, t_{3}\right)-u\left(\cdot, t_{2}\right)}{t_{3}-t_{2}}, \varphi\right)-\left(u\left(\cdot, t_{0}\right), \Delta \varphi\right)-\left(\Phi\left(\cdot, t_{0}\right), \varphi\right)\right| \\
& \leq 2\left(t_{1}-t_{0}\right) \sup _{t>s}\|\Phi(\cdot, t)\|_{q, \rho}\|\Delta \varphi\|_{q /(q-1), \rho^{\prime}} .
\end{aligned}
$$

Since $t_{1}<t_{0}$ can be taken arbitrarily close to $t_{0}$, we see that

$$
\left(\frac{d u}{d t}\left(\cdot, t_{0}\right), \varphi\right)=\left(\Delta u\left(\cdot, t_{0}\right)+\Phi\left(\cdot, t_{0}\right), \varphi\right)
$$

in the sense of distributions. Since $t_{0}>s$ is arbitrary, we conclude that $u(x, t)$ is a solution of (2.6) in the sense of distributions in $(s, \infty)$. The condition (2.7) follows from (2.14) and the fact

$$
\exp ((t-s) \Delta) u_{0} \rightarrow u_{0} * \text {-weakly in }\left(L^{r, \rho}\left(\mathbb{R}^{2}\right)+L^{2, \rho}\left(\mathbb{R}^{2}\right)\right)^{2}
$$

as $t \rightarrow s+0$. If $u_{0} \in\left(L^{r, \tilde{\rho}}\left(\mathbb{R}^{2}\right)\right)^{2}$ as well, the fact (2.8) follows from (2.13) and (2.14).

Next, suppose that $u(x, t)$ is the solution of (2.6)-(2.7). Put

$$
v(\cdot, t)=\exp ((t-s) \Delta) u_{0}+\int_{0}^{t-s} \exp (\tau \Delta) \Phi(\cdot, t-\tau) d \tau .
$$

Then $v(x, t)$ is also the solution of (2.6)-(2.7). Putting $w(x, t)=u(x, t)-v(x, t)$, we have $\frac{d w}{d t}(x, t)=0$ for all $t>s$ and $w(\cdot, t) \rightarrow 0$ as $t \rightarrow s+0$. It follows that $w(x, t) \equiv 0$, and hence $u(x, t)=v(x, t)$ is the solution of (2.5).

We turn to the proof of Assertion (ii). Suppose that $u(t, x)$ is defined as the limit (2.9). Fix $s \in \mathbb{R}$. Then, for $t>s$, we have

$$
\begin{aligned}
u(\cdot, t)= & \int_{0}^{t-s} \exp (\tau \Delta) \Phi(\cdot, t-\tau) d \tau \\
& +\exp ((t-s) \Delta) \lim _{T \rightarrow \infty} \int_{t-s}^{T} \exp ((\tau-t+s) \Delta) \Phi(\cdot, t-\tau) d \tau \\
= & \int_{0}^{t-s} \exp (\tau \Delta) \Phi(\cdot, t-\tau) d \tau \\
& +\exp ((t-s) \Delta) \lim _{T \rightarrow \infty} \int_{0}^{T-t+s} \exp (\tau \Delta) \Phi(\cdot, s-\tau) d \tau
\end{aligned}
$$

It follows that

$$
u(\cdot, t)=\int_{0}^{t-s} \exp (\tau \Delta) \Phi(\cdot, t-\tau) d \tau+\exp ((t-s) \Delta) u(\cdot, s) .
$$

This implies that $u(x, t)$ is the solution of (2.5) with $u_{0}(x)=u(x, s) \in\left(L^{r, \rho}\left(\mathbb{R}^{2}\right)\right)^{2}$. Hence Assertion (i) implies that $u(x, t)$ is a solution of (2.6). Since $s \in \mathbb{R}$ is arbitrary, $u(x, t)$ is a solution of (2.10).

On the other hand, suppose that $u(x, t)$ is a solution of (2.10). Then Assertion (i) implies that the equality (2.28) holds for every $s, t \in \mathbb{R}$ such that $s<t$. Fix $t \in \mathbb{R}$. Then we have 
$\exp ((t-s) \Delta) u(\cdot, s) \rightarrow 0 *$-weakly in $\left(L^{r, \rho}\left(\mathbb{R}^{2}\right)\right)^{2}$ as $s \rightarrow-\infty$. Indeed, suppose that $\varphi \in\left(L^{r /(r-1), \rho^{\prime}}\left(\mathbb{R}^{2}\right)\right)^{2}$. Then we have

$$
\begin{aligned}
|(\exp ((t-s) \Delta) u(\cdot, s), \varphi)| & =|(u(\cdot, s), \exp ((t-s) \Delta) \varphi)| \\
& \leq C\|u(\cdot, s)\|_{r, \rho}\|\exp ((t-s) \Delta) \varphi\|_{r /(r-1), \rho^{\prime}} .
\end{aligned}
$$

For every $\varepsilon>0$, there exists a function $\psi \in\left(L^{r /(r-1), \rho^{\prime}}\left(\mathbb{R}^{2}\right) \cap L^{1}\left(\mathbb{R}^{2}\right)\right)^{2}$ such that $\|\varphi-\psi\|_{r /(r-1), \rho^{\prime}}<\varepsilon$. From this fact it follows that

$$
\begin{aligned}
& \limsup _{s \rightarrow-\infty}\|\exp ((t-s) \Delta) \varphi\|_{r /(r-1), \rho^{\prime}} \\
& \leq \limsup _{s \rightarrow-\infty}\|\exp ((t-s) \Delta) \psi\|_{r /(r-1), \rho^{\prime}} \\
& \quad+\limsup _{s \rightarrow-\infty}\|\exp ((t-s) \Delta)(\varphi-\psi)\|_{r /(r-1), \rho^{\prime}} \\
& \leq 0+C \varepsilon=C \varepsilon .
\end{aligned}
$$

This implies that $\|\exp ((t-s) \Delta) \varphi\|_{r /(r-1), \rho^{\prime}} \rightarrow 0$ as $s \rightarrow-\infty$. On the other hand, the assumption implies that $\|u(\cdot, s)\|_{r, \rho}$ is bounded. Substituting these facts into (2.29) we see that $(\exp ((t-s) \Delta) u(\cdot, s), \varphi) \rightarrow 0$ as $s \rightarrow-\infty$. This implies the required weak-* convergence.

From this fact and (2.28) we see that

$$
\int_{0}^{t-s} \exp (\tau \Delta) \Phi(\cdot, t-\tau) d \tau=u(\cdot, t)-\exp ((t-s) \Delta) u(\cdot, s)
$$

converges $*$-weakly in $\left(L^{r, \rho}\left(\mathbb{R}^{2}\right)\right)^{2}$ to $u(\cdot, t)$ as $s \rightarrow-\infty$. This implies that $u(x, t)$ is the limit (2.9) in the sense of weak-* topology.

\section{Linear estimates}

We first have the following proposition on the weighted Lorentz boundedness.

Proposition 3.1 Suppose that either $-1 / 2<a \leq 0,1<p \leq 4$ or $0 \leq a<1 / 2,4 / 3 \leq$ $p<\infty$. Then there exists a positive constant $C$ depending only on a and $p$ such that the estimates

$$
\sup _{t>0} t^{k / 2}\left\|\langle\cdot\rangle^{a} \nabla^{k} \exp (t \Delta) f(\cdot)\right\|_{p, \tau} \leq C\left\|\langle\cdot\rangle^{a} f(\cdot)\right\|_{p, \tau}
$$

hold for $k=0,1,2,3, \tau \in[1, \infty]$ and for $f(x)$ such that $\langle\cdot\rangle^{a} f(\cdot) \in L^{p, \tau}$.

Proof For a homogeneous polynomial $q_{k}(\xi)$ of order $k$, regard $q_{k}(\xi) \exp \left(-t|\xi|^{2}\right)$ as a Fourier multiplier of Mikhlin type. Then we obtain the conclusion for $a=0$ from the $L^{p}$-boundedness and real interpolation.

We next consider the case $-1 / 2<a<0$ and $1<p \leq 4$. Choose $p_{0} \in(1, p)$, and put $p_{1}=8 /(1-2 a)$. Then we have $p_{0}<p \leq 4<p_{1}$,

$$
p_{j} a \geq \frac{8 a}{1-2 a}=-4+\frac{4}{1-2 a}>-2
$$


and $(2-a) p_{j}>2-a>2$, which implies $a p_{j}<2 p_{j}-2$ for $j=0,1$. Hence $\langle\cdot\rangle^{a p_{j}}$ is an $A_{p_{j}}$-weight for $j=0,1$. It follows that

$$
\begin{aligned}
& \sup _{t>0} t^{k / 2}\left\|\langle\cdot\rangle^{-a} \nabla^{k} \exp (t \Delta) f(\cdot)\right\|_{p_{j}}=\sup _{t>0} t^{k / 2}\left\|\nabla^{k} \exp (t \Delta) f(\cdot)\right\|_{L^{p_{j}}\left(\langle\cdot\rangle^{-a p_{j}} d x\right)} \\
& \leq C\|f\|_{L^{p_{j}\left(\langle\cdot\rangle^{-a p_{j}} d x\right)}}=C\left\|\langle\cdot\rangle^{-a} f\right\|_{p_{j}}
\end{aligned}
$$

for $j=0,1$. The conclusion follows from real interpolation.

We finally consider the case $0<a<1 / 2$ and $4 / 3 \leq p<\infty$. Put $p_{0}=8 /(7-2 a)$, and choose $p_{1} \in(p, \infty)$. Then we have $p_{0}<4 / 3 \leq p<p_{1}, p_{j} a>0>-2$ and

$$
(2-a) p_{j} \geq \frac{16-8 a}{7-2 a}=4-\frac{12}{7-2 a}>2,
$$

which implies $a p_{j}<2 p_{j}-2$ for $j=0,1$. In both cases $\langle\cdot\rangle^{a p_{j}}$ is an $A_{p_{j}}$-weight for $j=0,1$. The conclusion follows from these facts in the same way.

Next, the integral $I[G](x, s, t)$ satisfies the following propositions.

Proposition 3.2 Suppose that $0<a<1 / 2,2<p \leq 2 /(1-a)$ and that $1 \leq \rho \leq \infty$. Then, for every $T>0$, there exists a positive constant $C(T)$ independent of $G(x, t)$ such that, for every $\langle\cdot\rangle^{2 a} G(\cdot, t) \in B C\left(\mathbb{R},\left(L^{p / 2, \rho}\left(\mathbb{R}^{2}\right)\right)^{3}\right)$, the function $I_{0}[G](x, t, T)$ satisfies $\langle\cdot\rangle^{a} I_{0}[G](\cdot, t, T) \in B C\left(\mathbb{R},\left(L^{p, \rho}\left(\mathbb{R}^{2}\right)\right)^{2}\right)$ with the estimate

$$
\sup _{t \in \mathbb{R}}\left\|\langle\cdot\rangle^{a} I_{0}[G](\cdot, t, T)\right\|_{p, \rho} \leq C(T) \sup _{t \in \mathbb{R}}\left\|\langle\cdot\rangle^{2 a} G(\cdot, t)\right\|_{p / 2, \rho},
$$

with a positive constant $C(T)$ such that $C(T) \rightarrow 0$ as $T \rightarrow+0$, where

$$
\left\|\langle\cdot\rangle^{2 a} G(\cdot, t)\right\|_{p / 2, \rho}=\sum_{k=1}^{3}\left\|\langle\cdot\rangle^{2 a} G_{k}(\cdot, t)\right\|_{p / 2, \rho} .
$$

Proof There exist homogeneous polynomials $Q_{j, k}(\xi)$ of order 1 such that we have

$$
\begin{aligned}
I[G](x, s, t) & =\left(\sum_{k=1}^{3} \mathscr{F}^{-1}\left[\exp \left(-s|\xi|^{2}\right) Q_{j, k}(\xi) \mathscr{F}\left[G_{k}(\cdot, t-s)\right](\xi)\right]\right)_{j=1,2} \\
& =\left(\sum_{k=1}^{3} \int_{\mathbb{R}^{2}} K_{j, k}(x-y, s) G_{k}(y, t-s) d y\right)_{j=1,2}
\end{aligned}
$$

with $K_{j, k}(x, t)=\mathscr{F}^{-1}\left[\exp \left(-t|\xi|^{2}\right) Q_{j, k}(\xi)\right](x)$. Then we have

$$
\langle x\rangle^{a}|I[G](x, s, t)| \leq \sum_{j, k=1}^{2}\left(I_{1, j, k}+I_{2, j, k}\right),
$$

where

$$
I_{1, j, k}=\int_{\mathbb{R}^{2}}\langle x-y\rangle^{a}\left|K_{j, k}(x-y, s) G_{k}(y, t-s)\right| d y
$$

and

$$
I_{2, j, k}=\int_{\mathbb{R}^{2}}\langle y\rangle^{a}\left|K_{j, k}(x-y, s) G_{k}(y, t-s)\right| d y .
$$


Since $\langle x\rangle^{a} \leq 1+|x|^{a} \leq 1+\left(\left|x_{1}\right|+\left|x_{2}\right|\right)|x|^{a-1}$, we have

$$
\left\|I_{1, j, k}\right\|_{p, \rho} \leq\left\|I_{1, j, k, 1}\right\|_{p, \rho}+\left\|I_{1, j, k, 2}\right\|_{p, \rho},
$$

where

$$
\begin{aligned}
I_{1, j, k, 1} \leq & \sum_{\ell=1}^{2} \int_{\mathbb{R}^{2}} \frac{1}{|x-y|^{1-a}} \\
& \left|\left(x_{\ell}-y_{\ell}\right) \mathscr{F}^{-1}\left[Q_{j, k}(\xi) \exp \left(-s|\xi|^{2}\right)\right](x-y) G_{k}(y, t-s)\right| d y
\end{aligned}
$$

and

$$
I_{1, j, k, 2} \leq \int_{\mathbb{R}^{2}}\left|\mathscr{F}^{-1}\left[Q_{j, k}(\xi) \exp \left(-s|\xi|^{2}\right)\right](x-y) G_{k}(y, t-s)\right| d y
$$

Then we have

$$
\begin{aligned}
\left\|I_{1, j, k, 1}\right\|_{p, \rho} & =\sum_{\ell=1}^{2} \| \int_{\mathbb{R}^{2}} \frac{1}{|x-y|^{1-a}} \\
& \left|\mathscr{F}^{-1}\left[\frac{\partial}{\partial \xi_{\ell}}\left(Q_{j, k}(\xi) \exp \left(-s|\xi|^{2}\right)\right)\right](x-y) G_{k}(y, t-s)\right| d y \|_{p, \rho} \\
& \leq C \sum_{\ell=1}^{2}\left\|\frac{1}{|x|^{1-a}} \mathscr{F}^{-1}\left[\frac{R_{j, k, \ell}(\xi)}{|\xi|^{2}} \exp \left(-s|\xi|^{2}\right)\right](x)\right\|_{\alpha, \infty}\left\|G_{k}(\cdot, t-s)\right\|_{\beta, \rho},
\end{aligned}
$$

where $R_{j, k, \ell}(\xi)$ is a homogeneous polynomial of order 2 ,

$$
\frac{1}{\alpha}=\frac{3}{4}-\frac{1}{2 p} \text { and } \frac{1}{\beta}=\frac{1}{4}+\frac{3}{2 p} .
$$

In the same way we have

$$
\left\|I_{1, j, k, 2}\right\|_{p, \rho} \leq C\left\|\mathscr{F}^{-1}\left[Q_{j, k}(\xi) \exp \left(-s|\xi|^{2}\right)\right](x)\right\|_{\alpha, \infty}\left\|G_{k}(\cdot, t-s)\right\|_{\beta, \rho}
$$

and

$$
\left\|I_{2, j, k}\right\|_{p, \rho} \leq C\left\|\mathscr{F}^{-1}\left[Q_{j, k}(\xi) \exp \left(-s|\xi|^{2}\right)\right](x)\right\|_{\alpha, \infty}\left\|\langle\cdot\rangle^{a} G_{k}(\cdot, t-s)\right\|_{\beta, \rho} .
$$

We then have

$$
\left\|G_{k}(\cdot, t-s)\right\|_{\beta, \rho} \leq\left\|\langle\cdot\rangle^{a} G_{k}(\cdot, t-s)\right\|_{\beta, \rho} \leq C \sup _{t \in \mathbb{R}}\left\|\langle\cdot\rangle^{2 a} G_{k}(\cdot, t)\right\|_{p / 2, \rho}\left\|\langle x\rangle^{-a}\right\|_{\delta, \infty}
$$

where

$$
\frac{1}{\delta}=\frac{1}{\beta}-\frac{2}{p}=\frac{1}{4}-\frac{1}{2 p} .
$$

Since $(1-a) / 2 \leq 1 / p<1 / 2$, it follows that $0<1 / \delta \leq a / 4$. This implies $\left\|\langle x\rangle^{-a}\right\|_{\delta, \infty}<\infty$, from which we obtain

$$
\left\|G_{k}(\cdot, t-s)\right\|_{\beta, \rho} \leq\left\|\langle\cdot\rangle^{a} G_{k}(\cdot, t-s)\right\|_{\beta, \rho} \leq C \sup _{t \in \mathbb{R}}\left\|\langle\cdot\rangle^{2 a} G_{k}(\cdot, t)\right\|_{p / 2, \rho} .
$$


Next, by the boundedness of the Riesz transform on the space $L^{\gamma, \infty}$, we see that

$$
\begin{aligned}
& \left\|\frac{1}{|x|^{1-a}} \mathscr{F}^{-1}\left[\frac{R_{j, k, \ell}(\xi)}{|\xi|^{2}} \exp \left(-s|\xi|^{2}\right)\right](x)\right\|_{\alpha, \infty} \\
& \quad \leq C\left\|\frac{1}{|x|^{1-a}}\right\|_{2 /(1-a), \infty}\left\|\mathscr{F}^{-1}\left[\frac{R_{j, k, \ell}(\xi)}{|\xi|^{2}} \exp \left(-s|\xi|^{2}\right)\right](x)\right\|_{\gamma, \infty} \\
& \quad \leq C\left\|\frac{1}{|x|^{1-a}}\right\|_{2 /(1-a), \infty}\left\|\frac{1}{4 \pi s} \exp \left(-\frac{|x|^{2}}{4 s}\right)\right\|_{\gamma, \infty},
\end{aligned}
$$

where $\frac{1}{\gamma}=\frac{1}{\alpha}-\frac{1-a}{2}$. Since

$$
\left\|\frac{1}{4 \pi s} \exp \left(-\frac{|x|^{2}}{4 s}\right)\right\|_{1}=1 \text { and }\left\|\frac{1}{4 \pi s} \exp \left(-\frac{|x|^{2}}{4 s}\right)\right\|_{\infty}=\frac{1}{4 \pi s},
$$

real interpolation implies $\left\|\frac{1}{4 \pi s} \exp \left(-\frac{|x|^{2}}{4 s}\right)\right\|_{\gamma, \infty}=C s^{1 / \gamma-1}$. It follows from this estimate and the fact $1 /|x|^{1-\alpha} \in L^{2 /(1-\alpha), \infty}$ that

$$
\left\|\frac{1}{|x|^{1-a}} \mathscr{F}^{-1}\left[\frac{R_{j, k, \ell}(\xi)}{|\xi|^{2}} \exp \left(-s|\xi|^{2}\right)\right](x)\right\|_{\alpha, \infty} \leq C s^{1 / \gamma-1} .
$$

In the same way we have

$$
\left\|\mathscr{F}^{-1}\left[Q_{j, k}(\xi) \exp \left(-s|\xi|^{2}\right)\right](x)\right\|_{\alpha, \infty} \leq C s^{1 / \alpha-3 / 2} .
$$

Since $p>2$, it follows that

$$
\frac{1}{\alpha}-\frac{3}{2}=\frac{3}{4}-\frac{1}{2 p}-\frac{3}{2}>-\frac{3}{4}-\frac{1}{4}=-1
$$

In view of this inequality, the estimates (3.1)-(3.7) imply

$$
\leq C \sup _{t-T \leq r \leq T}\left\|\langle\cdot\rangle^{a} I[G](\cdot, s, t)\right\|_{p, \rho}\|(\cdot, r)\|_{p / 2, \rho}\left(s^{1 / \gamma-1}+s^{1 / \alpha-2 / 3}\right)
$$

for $s \in[0, T]$. It follows that

$$
\begin{aligned}
\left\|\langle\cdot\rangle^{a} I_{0}[G](\cdot, t, T)\right\|_{p, \rho} & \leq \int_{0}^{T}\left\|\langle\cdot\rangle^{a} I[G](\cdot, s, t)\right\|_{p, \rho} d s \\
& \leq C(T) \sup _{t \in \mathbb{R}}\left\|\langle\cdot\rangle^{2 a} G(\cdot, t)\right\|_{p / 2, \rho}
\end{aligned}
$$

for every $T>0$, where $C(T)=C_{1}\left(T^{1 / \gamma}+T^{1 / \alpha-1 / 2}\right)$. This implies

$$
\left\|\langle\cdot\rangle^{a} I_{0}[G](\cdot, t, t)\right\|_{p, \rho} \leq C(t) \sup _{t \geq 0}\left\|\langle\cdot\rangle^{2 a} G(\cdot, t)\right\|_{p / 2, \rho}
$$

with a function $C(t)$ independent of $G(x, t)$. Since $C(t) \rightarrow 0$ holds as $t \rightarrow+0$, this estimate implies that $\langle\cdot\rangle^{a} I_{0}[G](\cdot, t, t)$ is right continuous in $L^{p, \rho}\left(\mathbb{R}^{2}\right)$ at $t=0$.

In order to show the continuity of $\langle\cdot\rangle^{a} I_{0}[G](\cdot, t, T)$ in $L^{p, \rho}\left(\mathbb{R}^{2}\right)$ with respect to $t$, fix $t_{0}$ and $\varepsilon>0$. Since $\langle\cdot\rangle^{2 a} G(\cdot, t)$ is uniformly continuous on $\left[t_{0}-T-1, t_{0}+1\right]$ with values in $\left(L^{p / 2, \rho}\left(\mathbb{R}^{2}\right)\right)^{3}$, we can choose $\delta\left(t_{0}\right) \in(0,1)$ so small that, if $r_{0}, r_{1} \in\left[t_{0}-T-1, t_{0}+1\right]$ 
satisfies $\left|r_{1}-r_{0}\right|<\delta\left(t_{0}\right)$, then we have $\left\|\langle\cdot\rangle^{2 a}\left(G\left(\cdot, r_{1}\right)-G\left(\cdot, r_{0}\right)\right)\right\|_{p / 2, \rho}<\varepsilon / C(T)$. Then, for $t_{1} \in\left(t_{0}-\delta\left(t_{0}\right), t_{0}+\delta\left(t_{0}\right)\right)$ we have

$$
\begin{aligned}
& \left\|\langle\cdot\rangle^{a}\left(I_{0}[G]\left(\cdot, t_{1}, T\right)-I_{0}[G]\left(\cdot, t_{0}, T\right)\right)\right\|_{p, \rho} \\
& \quad \leq \int_{0}^{T}\left\|\langle\cdot\rangle^{a}\left(I[G]\left(\cdot, s, t_{1}\right)-I[G]\left(\cdot, s, t_{0}\right)\right)\right\|_{p, \rho} d s \\
& \quad \leq C_{1} \sup _{0 \leq s \leq T}\left\|\langle\cdot\rangle^{2 a}\left(G\left(\cdot, t_{1}-s\right)-G\left(\cdot, t_{0}-s\right)\right)\right\|_{p / 2, \rho} \int_{0}^{T}\left(s^{1 / \gamma-1}+s^{1 / \alpha-2 / 3}\right) d s \\
& \quad \leq C(T) \sup _{t_{0}-T \leq r \leq t_{0}}\left\|\langle\cdot\rangle^{2 a}\left(G\left(\cdot, r+t_{1}-t_{0}\right)-G(\cdot, r)\right)\right\|_{p / 2, \rho}<\varepsilon .
\end{aligned}
$$

This shows that $\langle\cdot\rangle^{a} I_{0}[G](\cdot, t, T)$ is continuous at $t=t_{0}$ with values in $\left(L^{p, \rho}\left(\mathbb{R}^{2}\right)\right)^{2}$. Since $t_{0} \in \mathbb{R}$ is arbitrary, we see that $\langle\cdot\rangle I_{0}[G](\cdot, t, T) \in B C\left(\mathbb{R},\left(L^{p, \rho}\left(\mathbb{R}^{2}\right)\right)^{2}\right)$. This completes the proof.

Proposition 3.3 Suppose that $0<a<1 / 2,2<p \leq 2 /(1-a)$ and $1 \leq \rho \leq \infty$. For every $\langle\cdot\rangle^{2 a} G(\cdot, t) \in B C\left((0, \infty),\left(L^{p / 2, \rho}\left(\mathbb{R}^{2}\right)\right)^{4}\right)$, the function $I_{0}[G](x, t, t)$ satisfies $\langle\cdot\rangle^{a} I_{0}[G](\cdot, 0,0)=0$ and $\langle\cdot\rangle^{a} I_{0}[G](\cdot, t, t) \in C\left([0, \infty),\left(L^{p, \rho}\left(\mathbb{R}^{2}\right)\right)^{2}\right)$.

Proof Fix positive numbers $t_{0}$ and $\varepsilon$ arbitrarily. Then, for every $t_{1} \in\left(t_{0}-\delta_{0}, t_{0}+\delta_{0}\right)$, where $\delta_{0} \in\left(0, t_{0} / 2\right)$ will be determined later, we have

$$
\begin{aligned}
I_{0}[G]\left(x, t_{1}, t_{1}\right)-I_{0}[G]\left(x, t_{0}, t_{0}\right) \\
=I_{0}[G]\left(x, t_{1}, t_{0}-\delta_{0}\right)+\int_{t_{0}-\delta_{0}}^{t_{1}} I[G]\left(x, s, t_{1}\right) d s-I_{0}[G]\left(x, t_{0}, t_{0}-\delta_{0}\right) \\
\quad-\int_{t_{0}-\delta_{0}}^{t_{0}} I[G]\left(x, s, t_{0}\right) d s .
\end{aligned}
$$

Since

$$
\begin{aligned}
\int_{t_{0}-\delta_{0}}^{t_{1}} I[G]\left(x, s, t_{1}\right) d s & =\int_{0}^{t_{1}-t_{0}+\delta_{0}} I\left[G\left(\cdot, \cdot+t_{0}-\delta_{0}\right)\right]\left(x, s, t_{1}-t_{0}+\delta_{0}\right) d s \\
& =I_{0}\left[G\left(\cdot, \cdot+t_{0}-\delta_{0}\right)\right]\left(x, t_{1}-t_{0}+\delta_{0}, t_{1}-t_{0}+\delta_{0}\right),
\end{aligned}
$$

the estimate (3.10) implies that

$$
\begin{aligned}
\left\|\langle\cdot\rangle^{a} \int_{t_{0}-\delta_{0}}^{t_{1}} I[G]\left(\cdot, s, t_{1}\right) d s\right\|_{p, \rho} & \leq C\left(t_{1}-t_{0}+\delta_{0}\right) \sup _{t \geq 0}\left\|\langle\cdot\rangle^{2 a} G(\cdot, t)\right\|_{p / 2, \rho} \\
& \leq C\left(2 \delta_{0}\right) \sup _{t \geq 0}\left\|\langle\cdot\rangle^{2 a} G(\cdot, t)\right\|_{p / 2, \rho},
\end{aligned}
$$

where $C\left(2 \delta_{0}\right)$ is a positive constant independent of $G(x, t)$. Hence we can take $\delta_{0}$ sufficiently small so that

$$
\left\|\langle\cdot\rangle^{a} \int_{t_{0}-\delta_{0}}^{t_{1}} I[G]\left(\cdot, s, t_{1}\right) d s\right\|_{p, \rho}<\frac{\varepsilon}{3} .
$$


Choosing $t_{1}=t_{0}$ in (3.13), we obtain

$$
\left\|\langle\cdot\rangle^{a} \int_{t_{0}-\delta_{0}}^{t_{0}} I[G]\left(\cdot, s, t_{0}\right) d s\right\|_{p, \rho}<\frac{\varepsilon}{3} .
$$

Finally, putting $T=t_{0}-\delta_{0}$ and replacing $\varepsilon$ by $\varepsilon / 3$, we see from (3.11) that there exists $\delta \in\left(0, \delta_{0}\right]$ such that $\left|t_{1}-t_{0}\right|<\delta$ implies

$$
\left\|\langle\cdot\rangle^{a}\left(I_{0}\left(\cdot, t_{1}, t_{0}-\delta_{0}\right)-I_{0}\left(\cdot, t_{0}, t_{0}-\delta_{0}\right)\right)\right\|_{p, \rho}<\frac{\varepsilon}{3} .
$$

Substituting (3.13)-(3.15) into (3.12), we conclude that

$$
\left\|\langle\cdot\rangle^{a}\left(I_{0}\left(\cdot, t_{1}, t_{1}\right)-I_{0}\left(\cdot, t_{0}, t_{0}\right)\right)\right\|_{p, \rho}<\varepsilon
$$

Since $t_{0}$ and $\varepsilon$ are arbitrary, this estimate implies $\langle\cdot\rangle^{a} I_{0}(\cdot, t, t) \in C\left((0, \infty),\left(L^{p, \rho}\left(\mathbb{R}^{2}\right)\right)^{2}\right)$. Next, putting $T=t$ in (3.9), we conclude

$$
\left\|\langle\cdot\rangle^{a} I_{0}[G](\cdot, t, t)\right\|_{p, \rho} \leq C(t) \sup _{t \in \mathbb{R}}\left\|\langle\cdot\rangle^{2 a} G(\cdot, t)\right\|_{p / 2, \rho} .
$$

Since $t \rightarrow+0$ implies $C(t) \rightarrow 0$, we see that $\langle\cdot\rangle^{a} I_{0}[G](\cdot, t, t) \rightarrow 0$ in $\left(L^{p, \rho}\left(\mathbb{R}^{2}\right)\right)^{2}$ as $t \rightarrow+0$. This fact proves the right continuity at $t=0$ if we put $\langle\cdot\rangle^{a} I_{0}[G](\cdot, 0,0)=0$. This completes the proof.

We next show the result on symmetry. Then we have the following proposition.

Proposition 3.4 In addition to the assumptions of Proposition 3.2, we assume that the functions $G_{k}(x, t)$ satisfy the equalities (0.5) and (0.6). Then we have

$$
I[G]\left(x^{\perp}, t, s\right)=(I[G](x, t, s))^{\perp}
$$

for every $x \in \mathbb{R}^{2}, t \in \mathbb{R}$ and $s>0$. 
Proof We have the equality

$$
\begin{aligned}
I[G]\left(x^{\perp}, s, t\right)= & \frac{1}{4 \pi s} \int_{\mathbb{R}^{2}} \exp \left(-\frac{\left(x^{\perp}-z^{\perp}\right)^{2}}{4 s}\right) P \\
& \left(\begin{array}{l}
+\frac{\partial G_{1}}{\partial x_{1}}\left(z^{\perp}, t-s\right)+\frac{\partial G_{2}}{\partial x_{2}}\left(z^{\perp}, t-s\right)-\frac{\partial G_{3}}{\partial x_{2}}\left(z^{\perp}, t-s\right) \\
-\frac{\partial G_{1}}{\partial x_{2}}\left(z^{\perp}, t-s\right)+\frac{\partial G_{2}}{\partial x_{1}}\left(z^{\perp}, t-s\right)+\frac{\partial G_{3}}{\partial x_{1}}\left(z^{\perp}, t-s\right)
\end{array}\right) d z \\
= & \frac{1}{4 \pi s} \int_{\mathbb{R}^{2}} \exp \left(-\frac{(x-z)^{2}}{4 s}\right) P \\
& \left(\begin{array}{l}
\left.-\frac{\partial}{\partial z_{2}} G_{1}\left(z^{\perp}, t-s\right)+\frac{\partial}{\partial z_{1}} G_{2}\left(z^{\perp}, t-s\right)-\frac{\partial}{\partial z_{1}} G_{3}\left(z^{\perp}, t-s\right)\right) \\
-\frac{\partial}{\partial z_{1}} G_{1}\left(z^{\perp}, t-s\right)-\frac{\partial}{\partial z_{2}} G_{2}\left(z^{\perp}, t-s\right)-\frac{\partial}{\partial z_{2}} G_{3}\left(z^{\perp}, t-s\right)
\end{array}\right) d z \\
= & \frac{1}{4 \pi s} \int_{\mathbb{R}^{2}} \exp \left(-\frac{(x-z)^{2}}{4 s}\right) P \\
& \left(\begin{array}{l}
+\frac{\partial G_{1}}{\partial x_{2}}(z, t-s)-\frac{\partial G_{2}}{\partial x_{1}}(z, t-s)-\frac{\partial G_{3}}{\partial x_{1}}(z, t-s) \\
+\frac{\partial G_{1}}{\partial x_{1}}(z, t-s)+\frac{\partial G_{2}}{\partial x_{2}}(z, t-s)-\frac{\partial G_{3}}{\partial x_{2}}(z, t-s)
\end{array}\right) d z \\
= & (I[G](x, s, t))^{\perp} .
\end{aligned}
$$

This completes the proof.

This proposition immediately yields the following corollary.

Corollary 3.5 In addition to the assumptions of Proposition 3.2, we assume that the functions $G_{k}(x, t)$ satisfy the equalities (0.5) and (0.6). Then, for every $t \in \mathbb{R}$ and every $T>0$, the functions $I_{0}[G](x, t, T)$ (and hence also $I_{0}[G](x, t, t)$ for $t>0$ ) satisfy the symmetry property $(\mathrm{C} 4 \mathrm{E})$.

We next show the convergence of $I_{0}[F](x, t, T)$ as $T \rightarrow \infty$ by employing the duality argument. Choose $q \in[2 /(1+a), 2)$ such that $1 / p+1 / q=1$, and choose $\rho^{\prime}-$ and $\rho^{\prime}$ as (1.5). Furthermore, choose $r \in[1 / a, \infty)$ such that $1 / r=2 / q-1$, so that $2 / p+1 / r=1$. Then we have the following lemma.

Lemma 3.6 Suppose that $0<a<1 / 2, k=0,1,2,1<\alpha \leq 4, \beta<\infty$ and that $0 \leq 2 / \alpha-2 / \beta \leq k$. Then there exists a positive constant $C$ such that, for every $t>0$ and every $\varphi(x) \in L^{p}\left(\langle x\rangle^{-a \alpha}\right)$ satisfying the symmetry condition

$$
\varphi(-x)=-\varphi(x)
$$

we have the estimate

$$
\left\|\langle x\rangle^{-a-k+2 / \alpha-2 / \beta} \nabla(E(t) \varphi)(x)\right\|_{\beta} \leq C t^{-(k+1) / 2}\left\|\langle x\rangle^{-a} \varphi(x)\right\|_{\alpha} .
$$

Proof Writing $x=(R \cos \theta, R \sin \theta)$, we have

$$
\nabla_{1}^{\prime} \varphi(x)=\frac{\partial \varphi}{\partial R}(R \cos \theta, R \sin \theta)=\cos \theta \frac{\partial \varphi}{\partial x_{1}}(x)+\sin \theta \frac{\partial \varphi}{\partial x_{2}}(x)
$$


and

$$
\nabla_{2}^{\prime} \varphi(x)=\frac{1}{R} \frac{\partial \varphi}{\partial \theta}(R \cos \theta, R \sin \theta)=-\sin \theta \frac{\partial \varphi}{\partial x_{1}}(x)+\cos \theta \frac{\partial \varphi}{\partial x_{2}}(x) .
$$

If $\varphi(x)$ satisfies (C2E), then we have $\nabla^{\prime} \varphi(x)=\left(\nabla_{1}^{\prime} \varphi(x), \nabla_{2}^{\prime} \varphi(x)\right)$ satisfies $(\mathrm{C} 2 \mathrm{E})$, and we have

$$
\left(\begin{array}{c}
\nabla_{1}^{\prime} \varphi(x) \\
\nabla_{2}^{\prime} \varphi(x)
\end{array}\right)=\left(\begin{array}{cc}
\cos \theta & \sin \theta \\
-\sin \theta & \cos \theta
\end{array}\right)\left(\begin{array}{l}
\nabla_{x_{1}} \varphi(x) \\
\nabla_{x_{2}} \varphi(x)
\end{array}\right)
$$

This implies $\left|\nabla^{\prime} \varphi(x)\right|=|\nabla \varphi(x)|$ for every $\left(x_{1}, x_{2}\right) \neq(0,0)$.

We first consider the case $k=0$. In this case we have $\alpha=\beta$. Applying Proposition 3.1 with $p=\tau=\alpha$ and $k=1$, we obtain

$$
\left\|\langle x\rangle^{-a} \nabla(E(t) \varphi)(x)\right\|_{\alpha} \leq C t^{-1 / 2}\left\|\langle x\rangle^{-a} \varphi\right\|_{\alpha},
$$

as required.

We next consider the case $k=1, r>0$ and $0 \leq 2 / \alpha-2 / \beta \leq 1$. Since $\nabla^{\prime}(E(t)) \varphi(x)$ satisfies (C2E), we have

$$
\int_{|x| \leq 1} \nabla^{\prime}(E(t)) \varphi(x) d x=0
$$

and

$$
\int_{2^{j-1} \leq|x| \leq 2^{j}} \nabla^{\prime}(E(t)) \varphi(x) d x=0 \text { for every } j=1,2, \ldots
$$

Hence we can apply Poincaré's inequality and the Sobolev embedding theorem to obtain

$$
\begin{aligned}
& \left\|\langle x\rangle^{-a-1+2 / \alpha-2 / \beta} \nabla(E(t) \varphi)(x)\right\|_{\beta} \\
& \leq\left(\left\|\nabla^{\prime}(E(t) \varphi)(x)\right\|_{L^{\beta}(\{x|| x \mid \leq 1\})}^{\beta}\right. \\
& \left.+\sum_{j=1}^{\infty}\left(2^{(-a-1+2 / \alpha-2 / \beta) j}\left\|\nabla^{\prime}(E(t) \varphi)(x)\right\|_{L^{\beta}\left(\left\{x\left|2^{j-1} \leq\right| x \mid \leq 2^{j}\right\}\right)}\right)^{\beta}\right)^{1 / \beta} \\
& \leq C\left(\left\|\nabla \nabla^{\prime}(E(t) \varphi)(x)\right\|_{L^{\alpha}(\{x|| x \mid \leq 1\})}^{\beta}\right. \\
& \left.+\sum_{j=1}^{\infty}\left(2^{-a j}\left\|\nabla \nabla^{\prime}(E(t) \varphi)(x)\right\|_{L^{\alpha}\left(\left\{x\left|2^{j-1} \leq\right| x \mid \leq 2^{j}\right\}\right)}\right)^{\beta}\right)^{1 / \beta} \\
& \leq C\left(\left\|\nabla \nabla^{\prime}(E(t) \varphi)(x)\right\|_{L^{\alpha}(\{x|| x \mid \leq 1\})}^{\alpha}\right. \\
& \left.+\sum_{j=1}^{\infty}\left(2^{-a j}\left\|\nabla \nabla^{\prime}(E(t) \varphi)(x)\right\|_{L^{\alpha}\left(\left\{x\left|2^{j-1} \leq\right| x \mid \leq 2^{j}\right\}\right)}\right)^{\alpha}\right)^{1 / \alpha} \\
& \leq C\left\|\langle x\rangle^{-a} \nabla^{2}(E(t) \varphi)(x)\right\|_{\alpha}
\end{aligned}
$$

From this estimate and Proposition 3.1 with $p=\tau=\alpha$ and $k=2$ we conclude that

$$
\left\|\langle x\rangle^{-a-1+2 / \alpha-2 / \beta} \nabla(E(t) \varphi)(x)\right\|_{\beta} \leq C t^{-1}\left\|\langle x\rangle^{-a} \varphi(x)\right\|_{\alpha},
$$

as required. 
We finally consider the case $k=2, r>0$ and $0 \leq 2 / \alpha-2 / \beta \leq 2$. If $2 / \alpha-2 / \beta \leq 1$, put $\gamma=\beta$. If $2 / \alpha-2 / \beta>1$, put $\gamma=2 \alpha /(2-\alpha)$. In both cases we have $0 \leq 2 / \gamma-2 / \beta \leq 1$. Hence (3.17) implies

$$
\left\|\langle x\rangle^{-a-2+2 / \alpha-2 / \beta} \nabla(E(t) \varphi)(x)\right\|_{\beta} \leq C\left\|\langle x\rangle^{-a-1+2 / \alpha-2 / \gamma} \nabla^{2}(E(t) \varphi)(x)\right\|_{\gamma} .
$$

Since $\nabla^{2}(E(t)) \varphi(x)$ satisfies $(\mathrm{C} 2 \mathrm{E})$, we can estimate

$$
\begin{aligned}
&\left\|\langle x\rangle^{-a-1+2 / \alpha-2 / \gamma} \nabla^{2}(E(t) \varphi)(x)\right\|_{\gamma} \\
&=\left(\left\|\nabla^{2}(E(t) \varphi)(x)\right\|_{L^{\gamma}(\{x|| x \mid \leq 1\})}^{\gamma}\right. \\
&\left.+\sum_{j=1}^{\infty}\left(2^{(-a-1+2 / \alpha-2 / \gamma) j}\left\|\nabla^{2}(E(t) \varphi)(x)\right\|_{L^{\gamma}\left(\left\{x\left|2^{j-1} \leq\right| x \mid \leq 2^{j}\right\}\right)}\right)^{\gamma}\right)^{1 / \gamma} \\
& \leq C\left(\left\|\nabla^{3}(E(t) \varphi)(x)\right\|_{L^{\alpha}(\{x|| x \mid \leq 1\})}^{\gamma}+\sum_{j=1}^{\infty}\left(2^{-a j}\left\|\nabla^{3}(E(t) \varphi)(x)\right\|_{L^{\alpha}\left(\left\{x\left|2^{j-1} \leq\right| x \mid \leq 2^{j}\right\}\right)}\right)^{\gamma}\right)^{1 / \gamma} \\
& \leq C\left(\left\|\nabla^{3}(E(t) \varphi)(x)\right\|_{L^{\alpha}(\{x|| x \mid \leq 1\})}^{\alpha}+\sum_{j=1}^{\infty}\left(2^{-a j}\left\|\nabla^{3}(E(t) \varphi)(x)\right\|_{L^{\alpha}\left(\left\{x\left|2^{j-1} \leq\right| x \mid \leq 2^{j}\right\}\right)}\right)^{\alpha}\right)^{1 / \alpha} \\
& \leq C\left\|\langle x\rangle^{-a} \nabla^{3}(E(t) \varphi)(x)\right\|_{\alpha}
\end{aligned}
$$

with some positive constant $C$. From this estimate, (3.18) and Proposition 3.1 with $p=\tau=\alpha$ and $k=3$ imply

$$
\left\|\langle x\rangle^{-a-2+2 / \alpha-2 / \beta} \nabla^{2}(E(t) \varphi)(x)\right\|_{\beta} \leq C t^{-3 / 2}\left\|\langle x\rangle^{-a} \varphi(x)\right\|_{\alpha},
$$

as required.

Corollary 3.7 Suppose that $0<a<1 / 2$ and that $1<q<4$. Suppose also that either $1 \leq \rho^{\prime}<\infty$ or $\rho=\infty$ - Then there exists a positive constant $C$ such that the following assertions hold.

(i) We have the estimate

$$
\left\|\langle x\rangle^{-2 a} \nabla(E(t) \varphi)(x)\right\|_{q, \rho^{\prime}} \leq C t^{-(1+a) / 2}\left\|\langle x\rangle^{-a} \varphi(x)\right\|_{q, \rho^{\prime}} .
$$

(ii) Put $s=2 q /(2-q)$. Then we can choose $C$ so that we have the estimate

$$
\left\|\langle x\rangle^{-2 a} \nabla(E(t) \varphi)(x)\right\|_{s, \rho^{\prime}} \leq C t^{-1-a / 2}\left\|\langle x\rangle^{-a} \varphi(x)\right\|_{q, \rho^{\prime}} .
$$

Proof We first prove Assertion (i). Lemma 3.6 for $k=0,1$ yields the estimate

$$
\begin{aligned}
\left\|\langle x\rangle^{-2 a} \nabla(E(t) \varphi)(x)\right\|_{q} & \leq\left\|\left\{\langle x\rangle^{-a} \nabla(E(t) \varphi)(x)\right\}^{1-a}\left\{\langle x\rangle^{-a-1} \nabla(E(t) \varphi)(x)\right\}^{a}\right\|_{q} \\
& \leq\left\|\langle x\rangle^{-a} \nabla(E(t) \varphi)(x)\right\|_{q}^{1-a}\left\|\langle x\rangle^{-a-1} \nabla(E(t) \varphi)(x)\right\|_{q}^{a} \\
& \leq C t^{-(1-a) / 2-a}\left\|\langle x\rangle^{-a} \varphi(x)\right\|_{q}=C t^{-(1+a) / 2}\left\|\langle x\rangle^{-a} \varphi(x)\right\|_{q}
\end{aligned}
$$

for $q \in$ (1.2). Assertion (i) follows from this estimate and real interpolation. 
We turn to the proof of Assertion (ii). Lemma 3.6 for $k=1,2$ implies

$$
\begin{aligned}
\left\|\langle x\rangle^{-2 a} \nabla(E(t) \varphi)(x)\right\|_{s} & \leq\left\|\left\{\langle x\rangle^{-a} \nabla(E(t) \varphi)(x)\right\}^{1-a}\left\{\langle x\rangle^{-a-1} \nabla(E(t) \varphi)(x)\right\}^{a}\right\|_{s} \\
& \leq\left\|\langle x\rangle^{-a} \nabla(E(t) \varphi)(x)\right\|_{s}^{1-a}\left\|\langle x\rangle^{-a-1} \nabla(E(t) \varphi)(x)\right\|_{s}^{a} \\
& \leq C t^{-(1-a)-3 a / 2}\left\|\langle x\rangle^{-a} \varphi(x)\right\|_{q} \leq C t^{-1-a / 2}\left\|\langle x\rangle^{-a} \varphi(x)\right\|_{q}
\end{aligned}
$$

for $q \in(1,2)$. Assertion (ii) follows from this estimate and real interpolation.

Proposition 3.8 Suppose that $0<a<1 / 2,2 /(1+a)<q<2$ and either $1 \leq \rho^{\prime}<\infty$ or $\rho^{\prime}=\infty-$. Put $r=q /(2-q)$. Then there exists a monotone-decreasing continuous function $\omega(S)$ on $[1, \infty)$ satisfying $\omega(S) \rightarrow 0$ as $S \rightarrow \infty$ such that, if $\varphi(x)$ satisfies $(\mathrm{C} 2 \mathrm{E})$ and $\langle x\rangle^{-a} \varphi(x) \in L^{q, \rho^{\prime}}\left(\mathbb{R}^{2}\right)$, then we have the estimate

$$
\int_{T}^{S}\left\|\langle x\rangle^{-2 a} \nabla(E(t) \varphi)(x)\right\|_{r, \rho^{\prime}} d t \leq(\omega(T)-\omega(S))\left\|\langle x\rangle^{-a} \varphi(x)\right\|_{q, \rho^{\prime}}
$$

for every $T, S$ such that $1 \leq T \leq S<\infty$.

Proof Put $s=2 q /(2-q)=2 r$. Then From Corollary 3.7 we obtain the estimates

$$
\left\|\langle x\rangle^{-2 a} \nabla(E(t) \varphi)(x)\right\|_{q, \rho^{\prime}} \leq C t^{-(1+a) / 2}\left\|\langle x\rangle^{-a} \varphi(x)\right\|_{q, \rho^{\prime}}
$$

and

$$
\left\|\langle x\rangle^{-2 a} \nabla(E(t) \varphi)(x)\right\|_{s, \rho^{\prime}} \leq C t^{-1-a / 2}\left\|\langle x\rangle^{-a} \varphi(x)\right\|_{q, \rho^{\prime}} .
$$

Put $\theta=2-2 / q$. Then we have $0<\theta<1$, and

$$
\frac{1-\theta}{q}+\frac{\theta}{s}=\frac{2-q}{q^{2}}+(2 q-2)\left(\frac{1}{q^{2}}-\frac{1}{2 q}\right)=\frac{1}{q}-1+\frac{1}{q}=\frac{1}{r}
$$

and

$$
(1-\theta) \frac{1+a}{2}+\theta\left(1+\frac{a}{2}\right)=\frac{a+1+\theta}{2}=\frac{a+1}{2}+1-\frac{1}{q} .
$$

Hence interpolation implies

$$
\left\|\langle x\rangle^{-2 a} \nabla(E(t) \varphi)(x)\right\|_{r, \rho^{\prime}} \leq C t^{-(1+a) / 2-1+1 / q}\left\|\langle x\rangle^{-a} \varphi(x)\right\|_{q, \rho^{\prime}} .
$$

Here we have $-(1+a) / 2+1 / q<0$. From this fact we see that the function $\omega(T)=$ $\frac{C T^{-(1+a) / 2+1 / q}}{(1+a) / 2-1 / q}$ is monotone-decreasing and continuous, and $\omega(T) \rightarrow 0$ as $T \rightarrow \infty$. Integrating (3.20) on $[T, S]$, we conclude (3.19).

We next give a proposition to treat the critical case.

Proposition 3.9 Suppose that $0<a<1 / 2$. Then there exists a positive constant $C$ such that, if $\varphi(x)$ satisfies $(\mathrm{C} 2 \mathrm{E})$ and $\langle x\rangle^{-a} \varphi(x) \in L^{2 /(1+a), 1}\left(\mathbb{R}^{2}\right)$, then we have the estimate

$$
\int_{0}^{\infty}\left\|\langle x\rangle^{-2 a} \nabla(E(t) \varphi)(x)\right\|_{1 / a, 1} d t \leq C\left\|\langle x\rangle^{-a} \varphi(x)\right\|_{2 /(1+a), 1} .
$$


Proof Put $p_{0}=4 /(2+3 a), p_{1}=4 /(2+a), \theta_{0}=a / 2$ and $\theta_{1}=3 a / 2$. Then we have $1<p_{0}<2 /(1+a)<p_{1}<2$ and $0<\theta_{0}<\theta_{1}<1$. Furthermore, we have

$$
\frac{\theta_{j}}{p_{j}}+\left(1-\theta_{j}\right)\left(\frac{1}{p_{j}}-\frac{1}{2}\right)=\frac{1}{p_{j}}+\frac{\theta_{j}-1}{2}=\frac{2+(3-2 j) a}{4}+\frac{(1+2 j) a-2}{4}=a
$$

and

$$
\theta_{j} \frac{1+a}{2}+\left(1-\theta_{j}\right)\left(1+\frac{a}{2}\right)=\frac{1+a}{2}+\frac{1-\theta_{j}}{2}=1+\frac{(1-2 j) a}{4}
$$

for $j=0,1$. Substituting these equality into the estimates

$$
\left\|\langle x\rangle^{-2 a} \nabla(E(t) \varphi)(x)\right\|_{p_{j}, 1} \leq C t^{-(1+a) / 2}\left\|\langle x\rangle^{-a} \varphi(x)\right\|_{p_{j}, 1}
$$

and

$$
\left\|\langle x\rangle^{-2 a} \nabla(E(t) \varphi)(x)\right\|_{2 p_{j} /\left(2-p_{j}\right), 1} \leq C t^{-(1+a) / 2}\left\|\langle x\rangle^{-a} \varphi(x)\right\|_{p_{j}, 1}
$$

which follow from Corollary 3.7 and applying real interpolation, we obtain

$$
\left\|\langle x\rangle^{-2 a} \nabla(E(t) \varphi)(x)\right\|_{1 / a, 1} \leq C t^{-1-(1-2 j) a / 4}\left\|\langle x\rangle^{-a} \varphi(x)\right\|_{p_{j}, 1}
$$

for $j=0,1$.

We now consider a mapping $f$ from $\mathbb{R}_{+}=(0, \infty)$ into itself by

$$
f(t)=\left\|\langle x\rangle^{-2 a} \nabla(E(t)) \varphi(x)\right\|_{1 / a, 1} .
$$

Then (3.21) implies that the mapping $\langle x\rangle^{-a} \varphi(x) \mapsto f(x)$ is bounded from $\left(L^{p_{j}, 1}\left(\mathbb{R}^{2}\right)\right)^{2}$ to $L^{1 /(1+(1-2 j) a / 4), \infty}((0, \infty))$ for $j=0,1$. From this fact and the interpolation equalities $\left(L^{p_{0}, 1}\left(\mathbb{R}^{2}\right), L^{p_{1}, 1}\left(\mathbb{R}^{2}\right)\right)_{1 / 2,1}=L^{2 /(1+a), 1}\left(\mathbb{R}^{2}\right)$ and

$$
\left(L^{1 /(1-a / 4), \infty}((0, \infty)), L^{1 /(1+a / 4), \infty}((0, \infty))\right)_{1 / 2,1}=L^{1,1}((0, \infty))=L^{1}((0, \infty)),
$$

we see that this mapping is bounded from $\left(L^{2 /(1+a), 1}\left(\mathbb{R}^{2}\right)\right)^{2}$ to $L^{1}((0, \infty))$; namely, the required estimate holds. This completes the proof.

We now prove the main results in this section.

Theorem 3.10 Suppose that $0<a<1 / 2,2<p<2 /(1-a)$ and $1 \leq \rho \leq \infty$. Then there exists a positive constant $C$ such that the following assumption holds. In addition to the assumption of Proposition 3.2, the functions $G_{1}(x, t), G_{2}(x, t)$ satisfy $(0.5)$ and the function $G_{3}(x, t)$ satisfies $(0.6)$. Then the limit $I_{1}[G](x, t)$ exists in the sense that, for every $t \in \mathbb{R}$, the function $\langle\cdot\rangle^{a} I_{0}[G](\cdot, t, T)$ converges to $\langle\cdot\rangle^{a} I_{1}[G](\cdot, t)$ in the weak-* topology of $\left(L^{p, \rho}\left(\mathbb{R}^{2}\right)\right)^{2}$. The limit $I_{1}[G](x, t)$ satisfies the symmetry property $(\mathrm{C} 4 \mathrm{E})$ for every $t \in \mathbb{R}$, and $\langle x\rangle^{a} I_{1}[G](\cdot, t)$ belongs to $B C\left(\mathbb{R},\left(L^{p, \rho}\left(\mathbb{R}^{2}\right)\right)^{2}\right)$ with the estimate

$$
\sup _{t \in \mathbb{R}}\left\|\langle\cdot\rangle^{a} I_{1}[G](\cdot, t)\right\|_{p, \rho} \leq C \sup _{t \in \mathbb{R}}\left\|\langle\cdot\rangle^{2 a} G(\cdot, t)\right\|_{p / 2, \rho} .
$$

Proof If we prove the convergence, the property (C4E) for $I_{0}[G](x, t, T)$ yields the property (C4E) for $I_{1}[G](x, t)$. We next put

$$
K(t, T)=\int_{\mathbb{R}^{2}} \psi(x)\langle x\rangle^{a} I_{0}[G](x, t, T) d x
$$


for $\psi(x)=\left(\psi_{1}(x), \psi_{2}(x)\right) \in\left(L^{q, \rho^{\prime}}\left(\mathbb{R}^{2}\right)\right)^{2}$.

We put $D_{ \pm}=\left\{x \in \mathbb{R}^{2} \mid \pm x_{1} \leq 0\right\}$ and $w(x, t)=\langle x\rangle^{a} I_{0}[G](x, t, T)$. Then, for every $\psi(x)$ as above, we have

$$
\int_{\mathbb{R}^{2}} w(x) \psi(x) d x=\int_{D_{+}} w(x) \psi(x) d x+\int_{D_{-}} w(x) \psi(x) d x .
$$

Since $w(x)$ satisfies $(\mathrm{C} 4 \mathrm{E})$, it follows that $w(x)$ satisfies $(\mathrm{C} 2 \mathrm{E})$. This implies

$$
\begin{aligned}
\int_{\mathbb{R}^{2}} w(x) \psi(x) d x & =\int_{D_{+}} w(x) \psi(x) d x-\int_{D_{+}} w(x) \psi(-x) d x \\
& =\int_{D_{+}} w(x)\{\psi(x)-\psi(-x)\} d x .
\end{aligned}
$$

In the same way we have

$$
\int_{\mathbb{R}^{2}} w(x) \psi(x) d x=\int_{D_{-}} w(x)\{\psi(x)-\psi(-x)\} d x .
$$

Putting

$$
\psi_{+}(x)=\frac{\psi(x)+\psi(-x)}{2}, \quad \psi_{-}(x)=\frac{\psi(x)-\psi(-x)}{2},
$$

we have $\psi_{-}(x)$ satisfies $(\mathrm{C} 2 \mathrm{E}),\left\|\psi_{-}\right\|_{q, \rho^{\prime}} \leq\|\psi\|_{q, \rho^{\prime}}$ and

$$
\int_{\mathbb{R}^{2}} w(x) \psi(x) d x=\int_{\mathbb{R}^{2}} w(x) \psi_{-}(x) d x .
$$

We next observe that $K(t, T)=K_{1}(t)+K_{2}(t, T)$, where

$$
K_{1}(t)=\int_{\mathbb{R}^{2}} \psi_{-}(x)\langle x\rangle^{a} \int_{0}^{1} I[G](x, s, t) d s d x
$$

and

$$
K_{2}(t, T)=\int_{\mathbb{R}^{2}} \psi_{-}(x)\langle x\rangle^{a} \int_{1}^{T} I[G](x, s, t) d s d x .
$$

Then Proposition 3.2 with $T=1$ implies that

$$
\begin{aligned}
&\left|K_{1}(t)\right| \leq C\left\|\psi_{-}\right\|_{q, \rho^{\prime}}\left\|\langle\cdot\rangle^{a} \int_{0}^{1} I[G](\cdot, s, t) d s\right\|_{p, \rho} \\
& \leq C\|\psi\|_{q, \rho^{\prime}} \sup _{t \in \mathbb{R}}\left\|\langle\cdot\rangle^{2 a} G(\cdot, t)\right\|_{p / 2, \rho} .
\end{aligned}
$$

It follows that

$$
\sup _{t \in \mathbb{R}}\left|K_{1}(t)\right| \leq C\|\psi\|_{q, \rho^{\prime}} \sup _{t \in \mathbb{R}}\left\|\langle\cdot\rangle^{2 a} G(\cdot, t)\right\|_{p / 2, \rho} .
$$

In order to estimate $K_{2}(t, T)$, we put $\varphi(x)=P\langle x\rangle^{a} \psi_{-}(x)$. Then $\varphi(x)$ satisfies (C2E). Furthermore, for every $\alpha \in(1,2 / a)$, the weight $\langle x\rangle^{-a \alpha}$ is an $A_{\alpha}$ weight. It follows that

$$
\left\|\langle x\rangle^{-a} \varphi(x)\right\|_{\alpha}=\left\|P\langle x\rangle^{a} \psi_{-}(x)\right\|_{L^{\alpha}\left(\langle x\rangle^{-a \alpha}\right)} \leq C\left\|\langle x\rangle^{a} \psi_{-}(x)\right\|_{L^{\alpha}\left(\langle x\rangle^{-a \alpha}\right)}=C\|\psi\|_{\alpha} .
$$

Hence we have

$$
\left\|\langle x\rangle^{-a} \varphi(x)\right\|_{\alpha, \rho^{\prime}} \leq C\|\psi\|_{\alpha, \rho^{\prime}}
$$


for $\alpha \in(1,2 / a)$ and every $\rho^{\prime}$ by real interpolation.

On the other hand, we have the equality

$$
\begin{aligned}
K_{2}(t, T)= & \int_{\mathbb{R}^{2}} \int_{1}^{T}\left(\begin{array}{l}
\varphi_{1}(x) \\
\varphi_{2}(x)
\end{array}\right) \int_{\mathbb{R}^{2}} \frac{1}{4 \pi s} \exp \left(-\frac{(x-y)^{2}}{4 s}\right) \\
& \left(\begin{array}{l}
\left.+\frac{\partial G_{1}}{\partial x_{1}}(y, t-s)+\frac{\partial G_{2}}{\partial x_{2}}(y, t-s)-\frac{\partial G_{3}}{\partial x_{2}}(y, t-s)\right) d y d s d x \\
-\frac{\partial G_{1}}{\partial x_{2}}(y, t-s)+\frac{\partial G_{2}}{\partial x_{1}}(y, t-s)+\frac{\partial G_{3}}{\partial x_{1}}(y, t-s)
\end{array}\right) \int_{\mathbb{R}^{2}} \exp \left(-\frac{(x-y)^{2}}{4 s}\right) \varphi_{1}(x) d x \\
= & \int_{\mathbb{R}^{2}} \int_{1}^{T} \frac{1}{4 \pi s}\left(G_{1}(y, t-s)\left\{-\frac{\partial}{\partial y_{1}} \int^{2}\right)\right. \\
& \left.+\frac{\partial}{\partial y_{2}} \int_{\mathbb{R}^{2}} \exp \left(-\frac{(x-y)^{2}}{4 s}\right) \varphi_{2}(x) d x\right\} \\
& +G_{2}(y, t-s)\left\{-\frac{\partial}{\partial y_{2}} \int_{\mathbb{R}^{2}} \exp \left(-\frac{(x-y)^{2}}{4 s}\right) \varphi_{1}(x) d x\right. \\
& \left.-\frac{\partial}{\partial y_{1}} \int_{\mathbb{R}^{2}} \exp \left(-\frac{(x-y)^{2}}{4 s}\right) \varphi_{2}(x) d x\right\} \\
& +G_{3}(y, t-s)\left\{\frac{\partial}{\partial y_{2}} \int_{\mathbb{R}^{2}} \exp \left(-\frac{(x-y)^{2}}{4 s}\right) \varphi_{1}(x) d x\right. \\
& \left.\left.+\frac{\partial}{\partial y_{1}} \int_{\mathbb{R}^{2}} \exp \left(-\frac{(x-y)^{2}}{4 s}\right) \varphi_{1}(x) d x\right\}\right) d s d y \\
= & \sum \sum_{k=1}^{3} \sum_{j=1}^{2} \int_{1}^{T}\left(G_{k}(\cdot, t-s), P_{j, k}(\nabla)\left(E(s) \varphi_{j}\right)(\cdot)\right) d s,
\end{aligned}
$$

where $P_{j, k}(\nabla)$ is a homogeneous differential operator of order 1 .

Then, for every $t \in \mathbb{R}$, Proposition 3.8 implies that

$$
\begin{aligned}
\left|K_{2}(t, S)-K_{2}(t, T)\right| & =\left|\sum_{k=1}^{3} \sum_{j=1}^{2} \int_{T}^{S}\left(G_{k}(\cdot, t-s), P_{j, k}(\nabla)\left(E(s) \varphi_{j}\right)(\cdot)\right) d s\right| \\
& \leq C \sum_{k=1}^{3} \int_{T}^{S} \sup _{t \in \mathbb{R}}\left\|\langle\cdot\rangle^{2 a} G_{k}(\cdot, t)\right\|_{p / 2, \rho}\left\|\langle\cdot\rangle^{-2 a} \nabla(E(s) \varphi)(x)\right\|_{r, \rho^{\prime}} d s \\
& \leq C \sup _{t \in \mathbb{R}}\left\|\langle\cdot\rangle^{2 a} G(\cdot, t)\right\|_{p / 2, \rho}(\omega(T)-\omega(S))\left\|\langle x\rangle^{-a} \varphi(x)\right\|_{q, \rho^{\prime}}
\end{aligned}
$$

for every $S, T$ such that $1 \leq T \leq S<\infty$. This estimate, together with (3.25), implies

$$
\left|K_{2}(t, S)-K_{2}(t, T)\right| \leq C \sup _{t \in \mathbb{R}}\left\|\langle\cdot\rangle^{2 a} G(\cdot, t)\right\|_{p / 2, \rho} \omega(T)\|\psi\|_{q, \rho^{\prime}} .
$$

Since the the right-hand side tends to 0 as $T \rightarrow \infty$, the limit $\tilde{K}_{2}(t)=\lim _{T \rightarrow \infty} K_{2}(t, T)$ exists and satisfies the estimate

$$
\left|\tilde{K}_{2}(t)-K_{2}(t, T)\right| \leq C \sup _{t \in \mathbb{R}}\left\|\langle\cdot\rangle^{2 a} G(\cdot, t)\right\|_{p / 2, \rho} \omega(T)\|\psi\|_{q, \rho^{\prime}} .
$$


Summing up this estimate and (3.24) we see that the limit $\tilde{K}(t)=\lim _{T \rightarrow \infty} K(t, T)$ exists and satisfies the estimates

$$
|\tilde{K}(t)-K(t, T)| \leq C \omega(T)\|\psi\|_{q, \rho^{\prime}} \sup _{t \in \mathbb{R}}\left\|\langle\cdot\rangle^{2 a} G(\cdot, t)\right\|_{p / 2, \rho}
$$

with a positive constant $C$. It follows that

$$
\begin{aligned}
\left\|\langle\cdot\rangle^{a}\left(I_{1}[G](\cdot, t)-I_{0}[G](\cdot, t, T)\right)\right\|_{p, \rho} & =\sup _{\psi \neq 0} \frac{|\tilde{K}(t)-K(t, T)|}{\|\psi\|_{q, \rho^{\prime}}} \\
& \leq C \omega(T)\|\psi\|_{q, \rho^{\prime}} \sup _{t \in \mathbb{R}}\left\|\langle\cdot\rangle^{2 a} G(\cdot, t)\right\|_{p / 2, \rho} .
\end{aligned}
$$

Since $t \in \mathbb{R}$ is arbitrary, we see that

$$
\begin{aligned}
& \sup _{t \in \mathbb{R}}\left\|\langle\cdot\rangle^{a}\left(I_{1}[G](\cdot, t)-I_{0}[G](\cdot, t, T)\right)\right\|_{p, \rho} \\
& \leq C \omega(T)\|\psi\|_{q, \rho^{\prime}} \sup _{t \in \mathbb{R}}\left\|\langle\cdot\rangle^{2 a} G(\cdot, t)\right\|_{p / 2, \rho} .
\end{aligned}
$$

This implies that $\langle\cdot\rangle^{a} I_{0}[G](\cdot, t, T)$ converges in $\left(L^{p, \rho}\left(\mathbb{R}^{2}\right)\right)^{2}$ as $T \rightarrow \infty$, and the convergence is uniform in $t \in \mathbb{R}$. Since $\langle\cdot\rangle^{a} I_{0}[G](\cdot, t, T) \in B C\left(\mathbb{R},\left(L^{p, \rho}\left(\mathbb{R}^{2}\right)\right)^{2}\right)$, the uniform convergence yields $\langle\cdot\rangle^{a} I_{1}[G](\cdot, t) \in B C\left(\mathbb{R},\left(L^{p, \rho}\left(\mathbb{R}^{2}\right)\right)^{2}\right)$.

For the critical case $p=2 /(1-a)$ we have the following theorem.

Theorem 3.11 Suppose that $0<a<1 / 2$. Then there exists a positive constant $C$ such that the following assertion holds. In addition to the assumption of Proposition 3.2 with $p=2 /(1-a)$ and $\rho=\infty$, the functions $G_{1}(x, t)$ and $G_{2}(x, t)$ satisfy $(0.5)$, and the function $G_{3}(x, t)$ satisfies (0.6). Then the limit $I_{1}[G](x, t)$ exists in the sense that, for every $t \in \mathbb{R}$, the function $\langle\cdot\rangle^{a} I_{0}[G](\cdot, t, T)$ converges to $\langle\cdot\rangle^{a} I_{1}[G](\cdot, t)$ with respect to the weak-* topology of $L^{p, \rho}\left(\mathbb{R}^{2}\right)$. The limit $I_{1}[G](x, t)$ satisfies the symmetry property $(\mathrm{C} 4 \mathrm{E})$, and the estimate

$$
\sup _{t \in \mathbb{R}}\left\|\langle\cdot\rangle^{a} I_{1}[G](\cdot, t)\right\|_{2 /(1-a), \infty} \leq C \sup _{t \in \mathbb{R}}\left\|\langle\cdot\rangle^{2 a} G(\cdot, t)\right\|_{1 /(1-a), \infty} .
$$

Proof We proceed in the same way as in the proof of Theorem 3.10. We can prove (3.24) in this case with $q=2 /(1+a), \rho^{\prime}=1, p=2 /(1-a)$ and $\rho=\infty$. On the other hand, we have

$$
\begin{aligned}
\left|K_{2}(t, S)-K_{2}(t, T)\right| & =\left|\sum_{k=1}^{3} \sum_{j=1}^{2} \int_{T}^{S}\left(G_{k}(\cdot, t-s), P_{j, k}(\nabla)\left(E(s) \varphi_{j}\right)(\cdot)\right) d s\right| \\
& \leq C \int_{T}^{S} \sup _{t \in \mathbb{R}}\left\|\langle\cdot\rangle^{2 a} G(\cdot, t)\right\|_{1 /(1-a), \infty}\left\|\langle\cdot\rangle^{-2 a} \nabla(E(s) \varphi)(x)\right\|_{1 / a, 1} d s \\
& =C \sup _{t \in \mathbb{R}}\left\|\langle\cdot\rangle^{2 a} G(\cdot, t)\right\|_{1 /(1-a), \infty} \int_{T}^{S}\left\|\langle\cdot\rangle^{-2 a} \nabla(E(s) \varphi)(x)\right\|_{1 / a, 1} d s
\end{aligned}
$$

for every $S, T$ such that $1 \leq T \leq S<\infty$. It follows that

$$
\begin{aligned}
& \sup _{t \in \mathbb{R}}\left|K_{2}(t, S)-K_{2}(t, T)\right| \\
& =C \sup _{t \in \mathbb{R}}\left\|\langle\cdot\rangle^{2 a} G(\cdot, t)\right\|_{1 /(1-a), \infty} \int_{T}^{\infty}\left\|\langle\cdot\rangle^{-2 a} \nabla(E(s) \varphi)(x)\right\|_{1 / a, 1} d s .
\end{aligned}
$$


Since the right-hand side tends to 0 as $T \rightarrow \infty$, the limit $\tilde{K}_{2}(t)=\lim _{T \rightarrow \infty} K_{2}(t, T)$ exists and satisfies the estimate

$$
\sup _{t \in \mathbb{R}}\left|K_{2}(t)\right| \leq C \sup _{t \in \mathbb{R}}\left\|\langle\cdot\rangle^{2 a} G(\cdot, t)\right\|_{1 /(1-a), \infty} \int_{1}^{\infty}\left\|\langle\cdot\rangle^{-2 a} \nabla(E(s) \varphi)(x)\right\|_{1 / a, 1} d s .
$$

Applying Proposition 3.9 together with (3.25), we obtain

$$
\sup _{t \in \mathbb{R}}\left|K_{2}(t)\right| \leq C \sup _{t \in \mathbb{R}}\left\|\langle\cdot\rangle^{2 a} G(\cdot, t)\right\|_{1 /(1-a), \infty}\|\psi\|_{2 /(1+a), 1} .
$$

Summing up this estimate and (3.24) we see that the limit $\tilde{K}(t)=\lim _{T \rightarrow \infty} K(t, T)$ exists and satisfies the estimate

$$
\sup _{t \in \mathbb{R}}|\tilde{K}(t)| \leq C \sup _{t \in \mathbb{R}}\left\|\langle\cdot\rangle^{2 a} G(\cdot, t)\right\|_{1 /(1-a), \infty}\|\psi\|_{2 /(1+a), 1} .
$$

Since $\psi \in\left(L^{2 /(1+a), 1}\left(\mathbb{R}^{2}\right)\right)^{2}$ is arbitrary, we conclude that $\langle\cdot\rangle^{a} I_{0}[G](\cdot, t, T)$ converges to $\langle\cdot\rangle^{a} I_{1}[G](\cdot, t)$ in the weak-* topology of $\left(L^{2 /(1-a), \infty}\left(\mathbb{R}^{2}\right)\right)^{2}$ as $T \rightarrow \infty$, together with the estimate

$$
\sup _{t \in \mathbb{R}}\left\|\langle\cdot\rangle^{a} I_{1}[G](\cdot, t)\right\|_{2 /(1-a), \infty} \leq C \sup _{t \in \mathbb{R}}\left\|\langle\cdot\rangle^{2 a} G(\cdot, t)\right\|_{1 /(1-a), \infty}
$$

with a positive constant $C$.

We finally show the continuity. Fix $t_{0} \in \mathbb{R}$, and let $t_{1}$ and $t_{2}$ be real numbers satisfying $t_{0}-1 / 2<t_{1} \leq t_{0} \leq t_{2}<t_{0}+1 / 2$ and $t_{1}<t_{2}$. Then we can write

$$
\begin{array}{r}
I_{0}[G]\left(x, t_{2}, T+t_{2}-t_{1}\right)-I_{0}[G]\left(x, t_{1}, T\right) \\
=J_{1}(x)+J_{2}(x)+J_{3}(x, T),
\end{array}
$$

where

$$
\begin{aligned}
J_{1}(x) & =\int_{0}^{1}\left\{I[G]\left(x, s, t_{2}\right)-I[G]\left(x, s, t_{1}\right)\right\} d s, \\
J_{2}(x) & =\int_{1}^{1+t_{2}-t_{1}} I[G]\left(x, s, t_{2}\right) d s, \\
J_{3}(x, T) & =\int_{1}^{T}\left\{I[G]\left(x, s+t_{2}-t_{1}, t_{2}\right)-I[G]\left(x, s, t_{1}\right)\right\} d s .
\end{aligned}
$$

Then we have

$$
I_{1}[G]\left(x, t_{2}\right)-I_{1}[G]\left(x, t_{1}\right)=J_{1}(x)+J_{2}(x)+\lim _{T \rightarrow \infty} J_{3}(x, T) .
$$

Since $J_{1}(x)=I_{0}[G]\left(x, t_{2}, 1\right)-I_{0}[G]\left(x, t_{1}, 1\right)$, Proposition 3.2 implies

$$
\langle\cdot\rangle^{a} J_{1}(\cdot) \rightarrow 0 \text { in }\left(L^{p, \rho}\left(\mathbb{R}^{2}\right)\right)^{2} \text { as } t_{1} \rightarrow t_{0}-0 \text { and } t_{2} \rightarrow t_{0}+0 .
$$

Next, (3.8) implies

$$
\left\|\langle\cdot\rangle^{a} J_{2}(\cdot)\right\|_{p, \rho} \leq C \sup _{t \in \mathbb{R}}\left\|\langle\cdot\rangle^{2 a} G(\cdot, t)\right\|_{p, \rho}\left\{\left(1+t_{2}-t_{1}\right)^{1 / \gamma}+\left(1+t_{2}-t_{1}\right)^{1 / \alpha+1 / 3}-2\right\} .
$$

It follows that

$$
\left\|\langle\cdot\rangle^{a} J_{2}(\cdot)\right\|_{p, \rho} \rightarrow 0 \text { as } t_{1} \rightarrow t_{0}-0 \text { and } t_{2} \rightarrow t_{0}+0
$$


We finally have

$$
\begin{aligned}
J_{3}(x, T)= & \int_{1+t_{2}-t_{1}}^{T+t_{2}-t_{1}} I[G]\left(x, s, t_{2}\right) d s-\int_{1}^{T} I[G]\left(x, s, t_{2}\right) d s \\
= & \int_{t_{1}-T}^{t_{1}-1} \exp \left(\left(t_{2}-s\right) \Delta\right)\left(\begin{array}{c}
\frac{\partial G_{1}}{\partial x_{1}}(\cdot, s)+\frac{\partial G_{2}}{\partial x_{2}}(\cdot, s)-\frac{\partial G_{3}}{\partial x_{2}}(\cdot, s) \\
-\frac{\partial G_{1}}{\partial x_{2}}(\cdot, s)+\frac{\partial G_{2}}{\partial x_{1}}(\cdot, s)+\frac{\partial G_{3}}{\partial x_{1}}(\cdot, s)
\end{array}\right) d s \\
& -\int_{t_{1}-T}^{t_{1}-1} \exp \left(-\left(t_{1}-s\right) \Delta\right)\left(\begin{array}{c}
\frac{\partial G_{1}}{\partial x_{1}}(\cdot, s)+\frac{\partial G_{2}}{\partial x_{2}}(\cdot, s)-\frac{\partial G_{3}}{\partial x_{2}}(\cdot, s) \\
-\frac{\partial G_{1}}{\partial x_{2}}(\cdot, s)+\frac{\partial G_{2}}{\partial x_{1}}(\cdot, s)+\frac{\partial G_{3}}{\partial x_{1}}(\cdot, s)
\end{array}\right) d s \\
= & \left\{\exp \left(-\left(t_{2}-t_{1}\right) \Delta\right)-1\right\} J_{4}(x, T),
\end{aligned}
$$

where

$$
J_{4}(x, T)=\int_{t_{1}-T}^{t_{1}-1} \exp \left(-\left(t_{1}-s\right) \Delta\right)\left(\begin{array}{c}
\frac{\partial G_{1}}{\partial x_{1}}(\cdot, s)+\frac{\partial G_{2}}{\partial x_{2}}(\cdot, s)-\frac{\partial G_{3}}{\partial x_{2}}(\cdot, s) \\
-\frac{\partial G_{1}}{\partial x_{2}}(\cdot, s)+\frac{\partial G_{2}}{\partial x_{1}}(\cdot, s)+\frac{\partial G_{3}}{\partial x_{1}}(\cdot, s)
\end{array}\right) d s
$$

Then we have

$$
\begin{aligned}
J_{4}(x, T) & =\int_{1}^{T} I[G]\left(x, s, t_{1}\right) d s=\exp (\Delta) \int_{0}^{T-1} I[G]\left(x, s, t_{1}-1\right) d s \\
& =\exp (\Delta) I_{0}[G]\left(x, t_{1}-1, T-1\right) .
\end{aligned}
$$

It follows that

$$
\lim _{T \rightarrow \infty} J_{3}(x, T)=\lim _{T \rightarrow \infty}\left\{\exp \left(\left(t_{2}-t_{1}\right) \Delta\right)-1\right\} \exp (\Delta) I_{0}[G]\left(x, t_{1}-1, T-1\right) .
$$

Since $\langle\cdot\rangle^{a} \exp (\Delta) I_{0}[G]\left(\cdot, t_{1}-1, T-1\right)$ converges in $\left(L^{p, \rho}\left(\mathbb{R}^{2}\right)\right)^{2}$ to $\langle\cdot\rangle^{a} \exp (\Delta) I_{1}[G]\left(\cdot, t_{1}-\right.$ 1) as $T \rightarrow \infty$, we see that the family

$$
\left\{\langle\cdot\rangle^{a} \exp (\Delta) I_{0}[G]\left(\cdot, t_{1}-1, T-1\right) \mid T \geq t_{0}+1, t_{0}-1 / 2<t_{1} \leq t_{0}\right\}
$$

is bounded in $\left(L^{p, \rho}\left(\mathbb{R}^{2}\right)\right)^{2}$. We next see that $\langle\cdot\rangle^{a p}$ is an $A_{2}$-weight. Hence the operator $\exp (\Delta)|\Delta|$ is bounded on $L^{p, \rho}\left(\mathbb{R}^{2}\right)$, and the family $\left\{\frac{|\Delta|^{-1}\{\exp (-r \Delta)-1\}}{r} \mid r>0\right\}$ is a family of bounded operators on $L^{p, \rho}\left(\mathbb{R}^{n}\right)$ whose operator norms are bounded. This implies

$$
\begin{aligned}
\langle\cdot\rangle^{a} J_{3}(\cdot, T) & \rightarrow\langle\cdot\rangle^{a}\left\{\exp \left(-\left(t_{2}-t_{1}\right) \Delta\right)-1\right\} \exp (\Delta) \lim _{T \rightarrow \infty} I_{0}[G]\left(\cdot, t_{1}-1, T-1\right) \\
& =\langle\cdot\rangle^{a}\left\{\exp \left(-\left(t_{2}-t_{1}\right) \Delta\right)-1\right\} \exp (\Delta) I_{1}[G]\left(\cdot, t_{1}-1\right)
\end{aligned}
$$

in $\left(L^{p, \rho}(\mathbb{R})\right)^{2}$ as $T \rightarrow \infty$, and the inequality

$$
\left\|\lim _{T \rightarrow \infty}\langle\cdot\rangle^{a} J_{3}(\cdot, T)\right\|_{p, \rho} \leq C\left(t_{2}-t_{1}\right)
$$

holds with a constant $C$. Substituting (3.31), (3.32) and the estimate above into (3.30), we see that $\langle\cdot\rangle^{a} I_{1}[G](\cdot, t)$ is continuous in $\left(L^{p, \rho}\left(\mathbb{R}^{2}\right)\right)^{2}$ at $t=t_{0}$. Since $t_{0} \in \mathbb{R}$ is arbitrary, we see that $\langle\cdot\rangle^{a} I_{1}[G](\cdot, t) \in B C\left(\mathbb{R},\left(L^{p, \rho}\left(\mathbb{R}^{2}\right)\right)^{2}\right)$. 
At the end of this section we show the boundedness of the solutions given in Proposition 3.3.

Theorem 3.12 Suppose that $\rho=\infty$ in the case $p=2 /(1-a)$. Then there exists a positive constant $C$ such that, in addition to the assumption of Proposition 3.3, we assume that the functions $G_{k}(x, t)$ satisfy the equalities (0.5) and (0.6). Then we have $\langle\cdot\rangle^{a} I_{0}[G](\cdot, t, t) \in$ $B C\left([0, \infty),\left(L^{p, \rho}\left(\mathbb{R}^{2}\right)\right)^{2}\right)$ with $\langle\cdot\rangle^{a} I_{0}[G](\cdot, 0,0)=0$ and the estimate

$$
\sup _{t \geq 0}\left\|\langle\cdot\rangle^{a} I_{0}[G](\cdot, t, t)\right\|_{p, \rho} \leq C \sup _{t \geq 0}\left\|\langle\cdot\rangle^{2 a} G(\cdot, t)\right\|_{p / 2, \rho} .
$$

Proof First, Proposition 3.2 implies

$$
\sup _{0 \leq t \leq 1}\left\|\langle\cdot\rangle^{a} I_{0}[G](\cdot, t, t)\right\|_{p, \rho} \leq C(1) \sup _{t \geq 0}\left\|\langle\cdot\rangle^{2 a} G(\cdot, t)\right\|_{p / 2, \rho} .
$$

Next, suppose that $t \geq 1$. Then we have

$$
\sup _{t \geq 1}\left\|\langle\cdot\rangle^{a} I_{0}[G](x, t, 1)\right\|_{p, \rho} \leq C(1) \sup _{t \geq 0}\left\|\langle\cdot\rangle^{2 a} G(\cdot, t)\right\|_{p / 2, \rho}
$$

and

$$
K_{2}(t, t)-K_{2}(t, 1)=\int_{\mathbb{R}^{2}} \psi(x)\langle x\rangle^{a}\left(I_{0}[G](x, t, t)-I_{0}[G](x, t, 1)\right) d x .
$$

If $2<p<2 /(1-a)$, the estimate (3.26) with $T=1$ implies

$$
\sup _{t \geq 1}\left|K_{2}(t, t)-K_{2}(t, 1)\right| \leq C \omega(1) \sup _{t \geq 1}\left\|\langle\cdot\rangle^{2 a} G(\cdot, t)\right\|_{p / 2, \rho}\|\psi\|_{q, \rho^{\prime}} .
$$

If $p=2 /(1-a)$ and $\rho=\infty$, the estimate (3.29) and Proposition 3.9 imply (3.35) with $p=2 /(1-a), q=2 /(1+a), \rho=\infty, \rho^{\prime}=1$ and $\omega(1)=1$.

Since $\psi \in\left(L^{q, \rho}\left(\mathbb{R}^{2}\right)\right)^{2}$ is arbitrary, we obtain

$$
\sup _{t \geq 1}\left\|\langle\cdot\rangle^{a}\left(I_{0}[F](\cdot, t, t)-I_{0}[F](\cdot, t, 1)\right)\right\|_{p, \rho} \leq C \omega(1) \sup _{t \geq 1}\left\|\langle\cdot\rangle^{2 a} F(\cdot, t)\right\|_{p / 2, \rho}
$$

in both cases. From this inequality and (3.34) we conclude that

$$
\sup _{t \geq 1}\left\|\langle\cdot\rangle^{a} I_{0}[G](\cdot, t, t)\right\|_{p, \rho} \leq(C(1)+C \omega(1)) \sup _{t \geq 1}\left\|\langle\cdot\rangle^{2 a} G(\cdot, t)\right\|_{p / 2, \rho} .
$$

This estimate and (3.33) imply the required boundedness.

\section{Proof of results}

We first prove Theorem 2.4. Define the function space $\mathscr{X}$ by

$$
\mathscr{X}=\left\{u(\cdot, t) \mid u(\cdot, t) \text { satisfies }(\mathrm{C} 4 \mathrm{E}),\langle\cdot\rangle^{a} u(\cdot, t) \in B C\left([0, \infty),\left(L^{p, \rho}\left(\mathbb{R}^{2}\right)\right)^{2}\right)\right\}
$$

equipped with the norm $\|u\|_{\mathscr{X}}=\sup _{t>0}\left\|\langle\cdot\rangle^{a} u(\cdot, t)\right\|_{p, \rho}$. Next, for $u_{0}(x)$ satisfying (C4E), for $F(x, t)$ such that $G_{1}[F](\cdot, t), G_{2}[F](\cdot, t)$ satisfying $(0.5), G_{3}[F](\cdot, t)$ satisfying $(0.6)$ and that $\left(\langle\cdot\rangle^{a} u_{0}(\cdot),\langle\cdot\rangle^{2 a} G[F](\cdot, t)\right) \in X$, and for $u(x, t) \in \mathscr{X}$, define $T\left[u_{0}, F, u\right]$ by the formula $T\left[u_{0}, F, u\right](x, t)=\exp (t \Delta) u_{0}(x)+I_{0}[H[F, u]](x, t, t)$. Then $T\left[u_{0}, F, u\right](x, t)$ satisfies $(\mathrm{C} 4 \mathrm{E})$, and the following proposition gives their estimates. 
Proposition 4.1 There exists a positive constant $C$ depending on $p$ and a such that, for every $u_{0}^{(j)}(x)$ satisfying $(\mathrm{C} 4 \mathrm{E}), G_{1}\left[F^{(j)}\right](\cdot, t), G_{2}\left[F^{(j)}\right](\cdot, t)$ satisfying $(0.5), G_{3}\left[F^{(j)}\right](\cdot, t)$ satisfying (0.6) such that $\left(\langle\cdot\rangle^{a} u_{0}^{(j)}(\cdot),\langle\cdot\rangle^{2 a} G\left[F^{(j)}\right](\cdot, t)\right) \in X$, and for $u^{(j)}(x, t) \in \mathscr{X}$ for $j=1,2$, we have the estimate

$$
\begin{aligned}
\| T & {\left[u_{0}^{(1)}, F^{(1)}, u^{(1)}\right]-T\left[u_{0}^{(2)}, F^{(2)}, u^{(2)}\right] \|_{\mathscr{X}} } \\
\leq & C\left\|\langle\cdot\rangle^{a}\left(u_{0}^{(1)}(\cdot)-u_{0}^{(2)}(\cdot)\right)\right\|_{p, \rho}+C \sup _{t>0}\left\|\langle\cdot\rangle^{2 a}\left(G\left[F^{(1)}\right](\cdot, t)-G\left[F^{(2)}\right](\cdot, t)\right)\right\|_{p / 2, \rho} \\
& +C\left(\left\|u^{(1)}\right\|_{\mathscr{X}}+\left\|u^{(2)}\right\|_{\mathscr{X}}\right)\left\|u^{(1)}-u^{(2)}\right\|_{\mathscr{X}} \cdot
\end{aligned}
$$

Proof In view of the equality $H[F, u](x, t)=G[F](x, t)+H[0, u](x, t)$, we obtain

$$
\begin{aligned}
\| T & {\left[u_{0}^{(1)}, F^{(1)}, u^{(1)}\right](t, x)-T\left[u_{0}^{(2)}, F^{(2)}, u^{(2)}\right] \|_{\mathscr{X}} } \\
\leq & \sup _{t>0}\left\|\langle\cdot\rangle^{a} \exp (t \Delta)\left(u_{0}^{(1)}-u_{0}^{(2)}\right)\right\|_{p, \rho}+\sup _{t>0}\left\|\langle\cdot\rangle^{a} I_{0}\left[G\left[F^{(1)}\right]-G\left[F^{(2)}\right], t, t\right]\right\|_{p, \rho} \\
& +\sup _{t>0}\left\|\langle\cdot\rangle^{a} I_{0}\left[H\left[0, u^{(1)}\right]-H\left[0, u^{(2)}\right], t, t\right]\right\|_{p, \rho} \\
\leq & C\left\|\langle\cdot\rangle^{a}\left(u_{0}^{(1)}-u_{0}^{(2)}\right)\right\|_{p, \rho}+C \sup _{t>0}\left\|\langle\cdot\rangle^{2 a}\left(G\left[F^{(1)}\right](\cdot, t)-G\left[F^{(2)}\right](\cdot, t)\right)\right\|_{p / 2, \rho} \\
& +C \sup _{t>0}\left\|\langle\cdot\rangle^{2 a}\left(H\left[0, u^{(1)}\right](\cdot, t)-H\left[0, u^{(2)}\right](\cdot, t)\right)\right\|_{p / 2, \rho} .
\end{aligned}
$$

with a positive constant $C$ by virtue of Proposition 3.1 and Theorem 3.12.

Since $H_{j}[0, u](x, t)$ is a linear combinations of products of the components of $u(x, t)$, we have

$$
\begin{aligned}
& \left\|\langle\cdot\rangle^{2 a}\left(H\left[0, u^{(1)}\right](\cdot, t)-H\left[0, u^{(2)}\right](\cdot, t)\right)\right\|_{p / 2, \rho} \\
& \leq \sum_{k, \ell=1}^{2}\left\|\langle\cdot\rangle^{2 a}\left(u_{k}^{(1)}(\cdot, t) u_{\ell}^{(1)}(\cdot, t)-u_{k}^{(2)}(\cdot, t) u_{\ell}^{(2)}(\cdot, t)\right)\right\|_{p / 2, \rho} \\
& \leq \sum_{k, \ell=1}^{2}\left(\left\|\langle\cdot\rangle^{a} u_{k}^{(1)}(\cdot, t)\right\|_{p, \rho}\left\|\langle\cdot\rangle^{a}\left(u_{\ell}^{(1)}(\cdot, t)-u_{\ell}^{(2)}(\cdot, t)\right)\right\|_{p, \rho}\right. \\
& \left.\quad+\left\|\langle\cdot\rangle^{a}\left(u_{k}^{(1)}(\cdot, t)-u_{k}^{(2)}(\cdot, t)\right)\right\|_{p \cdot \rho}\left\|\langle\cdot\rangle^{a} u_{\ell}^{(2)}(\cdot, t)\right\|_{p, \rho}\right) \\
& \leq C\left(\left\|\langle\cdot\rangle^{a} u^{(1)}(\cdot, t)\right\|_{p, \rho}+\left\|\langle\cdot\rangle^{a} u^{(2)}(\cdot, t)\right\|_{p, \rho}\right)\left\|\langle\cdot\rangle^{a}\left(u^{(1)}(\cdot, t)-u^{(2)}(\cdot, t)\right)\right\|_{p, \rho},
\end{aligned}
$$

which implies

$$
\begin{aligned}
& \sup _{t>0}\left\|\langle\cdot\rangle^{2 a}\left(H\left[0, u^{(1)}\right](\cdot, t)-H\left[0, u^{(2)}\right]\right)\right\|_{p / 2, \rho} \\
& \quad \leq C\left(\left\|u^{(1)}\right\|_{\mathscr{X}}+\left\|u^{(2)}\right\|_{\mathscr{X}}\right)\left\|u^{(1)}-u^{(2)}\right\|_{\mathscr{X}} .
\end{aligned}
$$

Substituting this estimate into (4.1) we obtain the conclusion. 
Proof of Theorem 2.4 Put $\varepsilon_{0}=1 / 4 C^{2}$. If $0<\varepsilon<\varepsilon_{0}$, the quadratic equation $C X^{2}-$ $X+C \varepsilon=0$ has distinct positive roots. Let $\omega(\varepsilon)$ denote the smaller one; namely, $\omega(\varepsilon)=\frac{1-\sqrt{1-4 C^{2} \varepsilon}}{2 C}$. For $\varepsilon=0$ and $\varepsilon=\varepsilon_{0}$ we define $\omega(\varepsilon)$ by the formula above.

Suppose that $\left\|\langle\cdot\rangle^{a} u_{0}(\cdot)\right\|_{p, \rho}+\sup _{t \in \mathbb{R}}\left\|\langle\cdot\rangle^{2 a} G[F](\cdot, t)\right\|_{p / 2, \rho} \leq \varepsilon<\varepsilon_{0}$ and that $u \in \mathscr{X}$ satisfies $\|u\|_{\mathscr{X}} \leq \omega(\varepsilon)$. Then Proposition 4.1 with $u_{0}^{(1)}=u_{0}, G\left[F^{(1)}\right]=G[F], u^{(1)}=u$, $u_{0}^{(2)}=0, G\left[F^{(2)}\right]=0$ and $u^{(2)}=0$ implies

$$
\left\|T\left[u_{0}, F, u\right]\right\|_{\mathscr{X}} \leq C \varepsilon+C \omega(\varepsilon)^{2}=\omega(\varepsilon) .
$$

Hence the mapping $T$ maps $B$ into itself, where $B$ denotes the closed ball in $\mathscr{X}$ with center 0 and radius $\omega(\varepsilon)$. Next, for $u^{(1)}, u^{(2)} \in B$, Proposition 4.1 with $u_{0}^{(1)}=u_{0}^{(2)}=u_{0}$ and $G\left[F^{(1)}\right]=G\left[F^{(2)}\right]=G[F]$ implies

$$
\begin{aligned}
& \| T {\left[u_{0}, F, u^{(1)}\right]-T\left[u_{0}, F, u^{(2)}\right] \|_{\mathscr{X}} } \\
& \quad \leq C\left(\left\|u^{(1)}\right\|_{\mathscr{X}}+\left\|u^{(2)}\right\|_{\mathscr{X}}\right)\left\|u^{(1)}-u^{(2)}\right\|_{\mathscr{X}} \leq 2 C \omega(\varepsilon)\left\|u^{(1)}-u^{(2)}\right\|_{\mathscr{X}} .
\end{aligned}
$$

Since $2 C \omega(\varepsilon)=1-\sqrt{1-4 C^{2} \varepsilon}<1$, the mapping $u \mapsto T\left[u_{0}, F, u\right]$ is a contraction mapping from $B$ into itself. Since $\mathscr{X}$ is complete, there uniquely exists a fixed point of $T$ in $B$. Furthermore, for every $R \in\left(0, \omega\left(\varepsilon_{0}\right)\right)$, we can choose $\varepsilon \in\left(0, \varepsilon_{0}\right)$ such that $\omega(\varepsilon)=R$. This implies the uniqueness of the fixed point $u$ such that $\|u\|_{\mathscr{X}}<\omega\left(\varepsilon_{0}\right)$. This is the required solution.

It remains only to show the uniform continuity. For $j=1,2$, let $\left(u_{0}^{(j)}(x), G\left[F^{(j)}\right](x, t)\right)$ satisfy the assumptions of the theorem with the estimate

$$
\left\|\langle\cdot\rangle^{a} u_{0}^{(j)}(\cdot)\right\|_{p, \rho}+\sup _{t>0}\left\|\langle\cdot\rangle^{2 a} G\left[F^{(j)}\right](\cdot, t)\right\|_{p / 2, \rho} \leq \varepsilon^{\prime}<\varepsilon_{0} .
$$

and that functions $u^{(j)}(x, t)$ are the fixed points of the mappings $u \mapsto T\left[u_{0}^{(j)}, G\left[F^{(j)}\right], u\right]$ respectively. Then Proposition 4.1 implies

$$
\begin{aligned}
\left\|u^{1}-u^{2}\right\|_{\mathscr{X}} \leq & C\left\|\langle\cdot\rangle^{a}\left(u_{0}^{(1)}(\cdot)-u_{0}^{(2)}(\cdot)\right)\right\|_{p, \rho} \\
& +C \sup _{t>0}\left\|\langle\cdot\rangle^{2 a}\left(G\left[F^{(1)}\right](\cdot, t)-G\left[F^{(2)}\right](\cdot, t)\right)\right\|_{p / 2, \rho} \\
& +C\left(\left\|u^{(1)}\right\|_{\mathscr{X}}+\left\|u^{(2)}\right\|_{\mathscr{X}}\right)\left\|u^{(1)}-u^{(2)}\right\|_{\mathscr{X}} \cdot
\end{aligned}
$$

Since $\left(\left\|u^{(1)}\right\|_{\mathscr{X}}+\left\|u^{(2)}\right\|_{\mathscr{X}}\right) \leq 2 C \omega\left(\varepsilon^{\prime}\right)<1$, we see that

$$
\begin{aligned}
& \left(1-2 C \omega\left(\varepsilon^{\prime}\right)\right)\left\|u^{(1)}-u^{(2)}\right\|_{\mathscr{X}} \\
& \quad \leq C\left(\left\|\langle\cdot\rangle^{a}\left(u_{0}^{(1)}(\cdot)-u_{0}^{(2)}(\cdot)\right)\right\|_{p, \rho}+\sup _{t>0}\left\|\langle\cdot\rangle^{2 a}\left(G\left[F^{(1)}\right]-G\left[F^{(2)}\right]\right)\right\|_{p / 2, \rho}\right) .
\end{aligned}
$$

This implies the required uniform continuity.

We can prove Theorem 2.3 in the same way as Theorem 2.4 with $u_{0}(x)=0$, by setting

$$
\mathscr{X}=\left\{u(\cdot, t) \mid u(\cdot, t) \text { satisfies }(\mathrm{C} 4 \mathrm{E}),\langle\cdot\rangle^{a} u(\cdot, t) \in B C\left(\mathbb{R},\left(L^{p, \rho}\left(\mathbb{R}^{2}\right)\right)^{2}\right)\right\}
$$


equipped with the norm $\|u\|_{\mathscr{X}}=\sup _{t \in \mathbb{R}}\left\|\langle\cdot\rangle^{a} u(\cdot, t)\right\|_{p, \rho}$ and making use of Theorem 3.10 or 3.11 in place of Theorem 3.12 and Proposition 3.1.

We turn to the proof of Corollary 1.2. Suppose that $F(x, t)$ is periodic with respect to $t$ with period $T$. Then we have

$$
\begin{aligned}
u(x, t+T) & =h[H[F, u]](x, t+T, \infty) \\
& =h[G[F]](x, t+T, \infty)+h[H[0, u]](x, t+T, \infty) \\
& =h[G[F]](x, t, \infty)+h[H[0, u(\cdot+T)]](x, t, \infty) .
\end{aligned}
$$

This implies that $v(x, t)=u(x, t+T)$ is another solution of (2.1) such that $\langle\cdot\rangle^{a} v(\cdot, t) \in$ $B C\left(\mathbb{R},\left(L^{p, \rho}\left(\mathbb{R}^{2}\right)\right)^{2}\right)$ with $\sup _{t \in \mathbb{R}}\left\|\langle\cdot\rangle^{a} v(\cdot, t)\right\|_{p, \rho}<\omega\left(\varepsilon_{0}\right)$. Hence we obtain $u(x, t+T)=$ $v(x, t)=u(x, t)$ by the uniqueness. This completes the proof of Assertion (i).

Next, suppose that $\langle\cdot\rangle^{2 a} G[F](\cdot, t)$ is almost periodic in $\left(L^{p / 2, \rho}\left(\mathbb{R}^{2}\right)\right)^{3}$ with the estimate $\sup _{t \in \mathbb{R}}\left\|\langle\cdot\rangle^{2 a} G[F](\cdot, t)\right\|_{p / 2, \rho}=\varepsilon^{\prime}<\varepsilon_{0}$, and let $\varepsilon$ be an arbitrary positive number. It follows

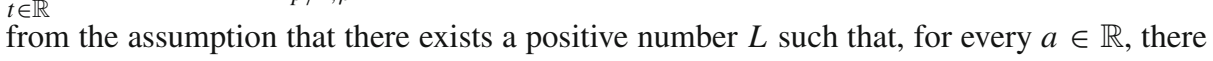
exists a number $T \in[a, a+L]$ such that

$$
\sup _{t \in \mathbb{R}}\left\|\langle\cdot\rangle^{2 a}(G[F](\cdot, t+T)-G[F](\cdot, t))\right\|_{p / 2, \rho}<\frac{\varepsilon}{C\left(\varepsilon^{\prime}\right)} .
$$

Then $u(x, t+T)$ is a solution corresponding to $G[F](x, t+T)$, and it satisfies the estimate

$$
\sup _{t \in \mathbb{R}}\left\|\langle\cdot\rangle^{a} u(\cdot, t+T)\right\|_{p, \rho}=\omega\left(\varepsilon^{\prime}\right)<\omega\left(\varepsilon_{0}\right) .
$$

Hence the estimate corresponding to (4.3) implies

$$
\|u(\cdot, t+T)-u(\cdot, t)\|_{\mathscr{X}} \leq C\left(\varepsilon^{\prime}\right) \sup _{t \in \mathbb{R}}\left\|\langle\cdot\rangle^{2 a}(G[F](\cdot, t+T)-G[F](\cdot, t))\right\|_{p / 2, \rho}<\varepsilon .
$$

That is, for every $\varepsilon>0$, there exists a positive number $L$ such that, for every $a \in \mathbb{R}$, there exists $T \in[a, a+L]$ satisfying $\|u(\cdot, t+T)-u(\cdot, t)\|_{\mathscr{X}}<\varepsilon$. Hence $\langle\cdot\rangle^{a} u(\cdot, t)$ is almost periodic in $\left(L^{p, \rho}\left(\mathbb{R}^{2}\right)\right)^{2}$. This completes the proof of Assertion (ii).

It remains only the proof of Theorem 2.5. First put

$$
L_{j}[v, w](x, t)=H_{j}[0, v+w](x, t)-H_{j}[0, v](x, t)-H_{j}[0, w](x, t)
$$

for $j=1,2$. Then the generalized Hölder inequality implies

$$
\left\|L_{j}[v, w](\cdot, t)\right\|_{2 p /(p+2), \infty} \leq C\|v(\cdot, t)\|_{2, \infty}\|w(\cdot, t)\|_{p, \infty}
$$

and

$$
\left\|H_{j}[0, w](\cdot, t)\right\|_{p / 2, \infty} \leq C\|w(\cdot, t)\|_{p, \infty}^{2}
$$

for $j=1,2$. Define the function space $\mathscr{Y}$ by

$$
\mathscr{Y}=\left\{u(\cdot, t) \in B C\left((0, \infty), L_{\sigma}^{2, \infty}\left(\mathbb{R}^{2}\right)\right) \mid t^{1 / 2-1 / p} u(\cdot, t) \in B C\left((0, \infty), L_{\sigma}^{p, \infty}\left(\mathbb{R}^{2}\right)\right)\right\}
$$

equipped with the norm

$$
\|u\|_{\mathscr{Y}}=\sup _{t>0}\|u(\cdot, t)\|_{2, \infty}+\sup _{t>0} t^{1 / 2-1 / p}\|u(\cdot, t)\|_{p, \infty},
$$


and put $S\left[w_{0}, w\right](x, t)=\exp (t \Delta) w_{0}(x)+S_{0}[w](x, t)$, where

$$
S_{0}[w](\cdot, t)=\int_{0}^{t} \exp ((t-\tau) \Delta) P\left(\begin{array}{cc}
\frac{\partial I_{1}[v, w]}{\partial x_{1}}(\cdot, \tau) & \frac{\partial I_{0}[v, w]}{\partial x_{2}}(\cdot, \tau, \tau) \\
-\frac{\partial I_{1}[v, w]}{\partial x_{2}}(\cdot, \tau) & \frac{\partial I_{0}[v, w]}{\partial x_{1}}(\cdot, \tau, \tau)
\end{array}\right) d \tau
$$

for $w(x, t) \in \mathscr{Y}$, where $I_{j}[v, w](x, \tau)=H_{j}[0, w](x, \tau)+L_{j}[v, w](x, \tau)$ for $j=1,2$. Then we have the following proposition.

Proposition 4.2 There exists a positive constant $C$ depending on $p$ such that, for every $w_{0}^{(1)}(x), w_{0}^{(2)}(x) \in L_{\sigma}^{2, \infty}(\Omega)$ and every $w_{1}(x, t), w_{2}(x, t) \in \mathscr{Y}$, we have $S\left[w_{0}^{(1)}, w_{1}\right](z, r)$, $S\left[w_{0}^{(2)}, w_{2}\right](x, t) \in \mathscr{Y}$ with the estimate

$$
\begin{aligned}
\left\|S\left[w_{0}^{(1)}, w_{1}\right]-S\left[w_{0}^{(2)}, w_{2}\right]\right\|_{\mathscr{Y}} \leq & C\left\|w_{0}^{(1)}-w_{0}^{(2)}\right\|_{2, \infty} \\
& +C\left(\left\|w_{1}\right\|_{\mathscr{Y}}+\left\|w_{2}\right\|_{\mathscr{Y}}+\sup _{t>0}\|v(\cdot, t)\|_{2, \infty}\right)\left\|w_{1}-w_{2}\right\|_{\mathscr{Y}} .
\end{aligned}
$$

Admitting this proposition for the moment, we give a proof of Theorem 2.5. We first put $K=1 / C$. Then, for $M<K$ and $0<\delta \leq \delta_{0}=\frac{(1-C M)^{2}}{4 C^{2}}$, the quadratic equation $x=C x^{2}+C M x+C \delta$ has positive roots. Let $\psi(\delta)$ denote the smaller one: namely,

$$
\psi(\delta)=\frac{1-C M-\sqrt{(1-C M)^{2}-4 C^{2} \delta}}{2 C} .
$$

Suppose that $\sup _{t>0}\|v(\cdot, t)\|_{2, \infty}=M$ and that $\left\|w_{0}\right\|_{2, \infty} \leq \delta<\delta_{0}$. If $\|w\|_{\mathscr{Y}} \leq \psi(\delta)$, then Proposition 4.2 with $w_{0}^{(1)}=w_{0}, w_{1}=w, w_{0}^{(2)}=0$ and $w_{2}=0$ implies that

$$
\left\|S\left[w_{0}, w\right]\right\|_{\mathscr{Y}} \leq C(\psi(\delta))^{2}+C M \psi(\delta)+C \delta=\psi(\delta) .
$$

Furthermore, if $\left\|w_{1}\right\|_{\mathscr{Y}},\left\|w_{2}\right\|_{\mathscr{Y}} \leq \psi(\delta)$, then Proposition 4.2 with $w_{0}^{(1)}=w_{0}^{(2)}=w_{0}$ implies that

$$
\begin{aligned}
& \left\|S\left[w_{0}, w_{1}\right]-S\left[w_{0}, w_{2}\right]\right\|_{\mathscr{Y}} \\
& \quad \leq(2 C \psi(\delta)+C M)\left\|w_{1}-w_{2}\right\|_{\mathscr{Y}}=\left(1-\sqrt{(1-C M)^{2}-4 C \delta}\right)\left\|w_{1}-w_{2}\right\|_{\mathscr{Y}} .
\end{aligned}
$$

Hence the mapping $w \mapsto S\left[w_{0}, w\right]$ is a contraction mapping from a closed ball in $\mathscr{Y}$ with center 0 and radius $\psi(\delta)$, and hence there exists a unique fixed point in this closed ball. For every $R \in\left(0, \psi\left(\delta_{0}\right)\right)$, we can choose $\delta \in\left(0, \delta_{0}\right)$ such that $\psi(\delta)=R$, which implies the uniqueness of the fixed point $w$ with $\|w\|_{\mathscr{Y}}<\psi\left(\delta_{0}\right)$. This $w$ is the required solution.

It remains only to show the uniform continuity. Suppose that $w_{0}^{(1)}(x), w_{0}^{(2)}(x) \in L_{\sigma}^{2, \infty}\left(\mathbb{R}^{2}\right)$ such that

$$
\max \left\{\left\|w_{0}^{(1)}\right\|_{2, \infty},\left\|w_{0}^{(2)}\right\|_{2, \infty}\right\} \leq \delta^{\prime}<\delta_{0},
$$

and let $w_{j}$ denote the fixed point of the mapping $w \mapsto S\left[w_{0}^{(j)}, w\right]$ respectively for $j=1,2$. Then Proposition 4.2 implies that

$$
\left\|w_{1}-w_{2}\right\|_{\mathscr{Y}} \leq C\left(\left\|w_{1}\right\|_{\mathscr{Y}}+\left\|w_{2}\right\|_{\mathscr{Y}}+M\right)\left\|w_{1}-w_{2}\right\|_{\mathscr{Y}}+C\left\|w_{0}^{(1)}-w_{0}^{(2)}\right\|_{2, \infty} .
$$


Since $\left\|w_{j}\right\|_{\mathscr{Y}} \leq \psi\left(\delta^{\prime}\right)$ for $j=1,2$, we conclude that

$$
\left(1-2 C \psi\left(\delta^{\prime}\right)-C M\right)\left\|w_{1}-w_{2}\right\|_{\mathscr{Y}} \leq C\left\|w_{0}^{(1)}-w_{0}^{(2)}\right\|_{2, \infty} .
$$

This implies the required uniform continuity.

Proof of Proposition 4.2 By the standard $L^{p}-L^{q}$ estimate and real interpolation we have

$$
\left\|\exp (t \Delta) w_{0}^{(1)}(\cdot)-\exp (t \Delta) w_{0}^{(2)}(\cdot)\right\|_{r, \infty} \leq C t^{1 / r-1 / 2}\left\|w_{0}^{(1)}-w_{0}^{(2)}\right\|_{2, \infty}
$$

for $r \in[2, p]$ with a constant $C$ depending only on $p \in(2, \infty)$. Next, put $w(x, t)=$ $w_{1}(x, t)-w_{2}(x, t)$. Then we have

$$
\left\|H_{j}[0, w](\cdot, t)\right\|_{p / 2, \infty} \leq C t^{2 / p-1}\left(\left\|w_{1}\right\|_{\mathscr{Y}}+\left\|w_{2}\right\|_{\mathscr{Y}}\right)\|w\|_{\mathscr{Y}}
$$

in the same way as (4.2). It follows that

$$
\begin{aligned}
\left\|S_{0}[w](\cdot, t)\right\|_{2, \infty} \leq & C \sum_{j=1}^{2} \int_{0}^{t}\left\{(t-\tau)^{-1 / 2-2 / p+1 / 2}\left\|H_{j}[0, w](\cdot, \tau)\right\|_{p / 2, \infty}\right. \\
& \left.+(t-\tau)^{-1 / 2-(1 / p+1 / 2)+1 / 2}\left\|L_{j}[v, w](\cdot, \tau)\right\|_{2 p /(2+p), \infty}\right\} d \tau \\
\leq & C \int_{0}^{t}\left\{(t-\tau)^{-2 / p} \tau^{2 / p-1}\left(\left\|w_{1}\right\|_{\mathscr{Y}}+\left\|w_{2}\right\|_{\mathscr{Y}}\right)\|w\|_{\mathscr{Y}}^{2}\right. \\
& \left.+(t-\tau)^{-1 / p-1 / 2}\|v(\cdot, \tau)\|_{2, \infty}\|w(\cdot, \tau)\|_{p, \infty}\right\} d \tau \\
\leq & C \int_{0}^{t}\left\{(t-\tau)^{-2 / p} \tau^{2 / p-1}\left(\left\|w_{1}\right\|_{\mathscr{Y}}+\left\|w_{2}\right\|_{\mathscr{Y}}\right)\|w\|_{\mathscr{Y}}^{2}\right. \\
& \left.+(t-\tau)^{-1 / p-1 / 2} \sup _{t>0}\|v(\cdot, t)\|_{2, \infty} \tau^{1 / p-1 / 2}\|w\|_{\mathscr{Y}}\right\} d \tau .
\end{aligned}
$$

We thus conclude

$$
\left\|S_{0}[w](t)\right\|_{2, \infty} \leq C\|w\|_{\mathscr{Y}}\left(\left\|w_{1}\right\|_{\mathscr{Y}}+\left\|w_{2}\right\|_{\mathscr{Y}}+\sup _{t>0}\|v(\cdot, t)\|_{2, \infty}\right) .
$$

In order to estimate $\left\|S_{0}[w](t)\right\|_{p, \infty}$, we employ the duality argument. For $\varphi(x) \in C_{0, \sigma}^{\infty}\left(\mathbb{R}^{2}\right)$, we have the duality

$$
\begin{aligned}
\left(S_{0}[w](\cdot, t), \varphi(\cdot)\right) & =\left(\int_{0}^{t} \exp ((t-\tau) \Delta) P\left(\begin{array}{cc}
\frac{\partial I_{1}[v, w]}{\partial x_{1}}(\cdot, \tau) & \frac{\partial I_{0}[v, w]}{\partial x_{2}}(\cdot, \tau, \tau) \\
-\frac{\partial I_{1}[v, w]}{\partial x_{2}}(\cdot, \tau) & \frac{\partial I_{0}[v, w]}{\partial x_{1}}(\cdot, \tau, \tau)
\end{array}\right) d \tau, \varphi\right) \\
& =\int_{0}^{t}\left(\left(\begin{array}{cc}
\frac{\partial I_{1}[v, w]}{\partial x_{1}}(\cdot, \tau) & \frac{\partial I_{0}[v, w]}{\partial x_{2}}(\cdot, \tau, \tau) \\
-\frac{\partial I_{1}[v, w]}{\partial x_{2}}(\cdot, \tau) & \frac{\partial I_{0}[v, w]}{\partial x_{1}}(\cdot, \tau, \tau)
\end{array}\right), \exp ((t-\tau) \Delta) \varphi\right) d \tau
\end{aligned}
$$


Since $C_{0, \sigma}^{\infty}(\Omega)$ is dense in $L_{\sigma}^{p /(p-1), 1}\left(\mathbb{R}^{2}\right)$, we can estimate

$$
\begin{aligned}
& \left\|S_{0}[w](\cdot, t)\right\|_{p, \infty} \\
& \leq \sup _{\varphi}\left|\int_{0}^{t}\left(\left(\begin{array}{cc}
\frac{\partial I_{1}[v, w]}{\partial x_{1}}(\cdot, \tau) & \frac{\partial I_{0}[v, w]}{\partial x_{2}}(\cdot, \tau, \tau) \\
-\frac{\partial I_{1}[v, w]}{\partial x_{2}}(\cdot, \tau) & \frac{\partial I_{0}[v, w]}{\partial x_{1}}(\cdot, \tau, \tau)
\end{array}\right), \exp ((t-\tau) \Delta) \varphi\right) d \tau\right| \\
& \leq C \sup _{\varphi} \int_{0}^{t}\left\{\left|\left(H_{j}[0, w](\cdot, \tau), \nabla \exp ((t-\tau) \Delta) \varphi\right)\right|\right. \\
& \left.\quad+\left|\left(L_{j}[v, w](\cdot, \tau), \nabla \exp ((t-\tau) \Delta) \varphi\right)\right|\right\} d \tau
\end{aligned}
$$

where the supremum is taken for $\varphi \in C_{0, \sigma}^{\infty}(\Omega)$ with $\|\varphi\|_{p /(p-1), 1} \leq 1$. It follows that $\left\|S_{0}[w](\cdot, t)\right\|_{p, \infty} \leq I_{1}+I_{2}+I_{3}$, where

$$
\begin{aligned}
& I_{1}=C \sup _{\varphi} \int_{0}^{t}\left\|H_{j}[0, w](\cdot, \tau)\right\|_{p / 2, \infty}\|\nabla \exp ((t-\tau) \Delta) \varphi\|_{p /(p-2), 1} d \tau, \\
& I_{2}=C \sup _{\varphi} \int_{0}^{t / 2}\left\|L_{j}[v, w](\cdot, \tau)\right\|_{2 p /(2+p), \infty}\|\nabla \exp ((t-\tau) \Delta) \varphi\|_{2 p /(p-2), 1} d \tau, \\
& I_{3}=C \sup _{\varphi} \int_{t / 2}^{t}\left\|L_{j}[v, w](\cdot, \tau)\right\|_{2 p /(2+p), \infty}\|\nabla \exp ((t-\tau) \Delta) \varphi\|_{2 p /(p-2), 1} d \tau .
\end{aligned}
$$

Employing (4.5) and the fact $\|\varphi\|_{p /(p-1), 1} \leq 1$, we can estimate $I_{1}$ as

$$
\begin{aligned}
I_{1} \leq & C \sup _{\varphi} \int_{0}^{t}\left(\left\|w_{1}\right\|_{\mathscr{Y}}+\left\|w_{2}\right\|_{\mathscr{Y}}\right)\|w\|_{\mathscr{Y}} \tau^{2 / p-1} \\
& (t-\tau)^{-1 / 2-(p-1) / p+(p-2) / p} d \tau \\
\leq & C t^{1 / p-1 / 2}\left(\left\|w_{1}\right\|_{\mathscr{Y}}+\left\|w_{2}\right\|_{\mathscr{Y}}\right)\|w\|_{\mathscr{Y}} .
\end{aligned}
$$

In the same way we can estimate $I_{2}$ as

$$
\begin{aligned}
I_{2} \leq & C \sup _{\varphi} \int_{0}^{t / 2}\|w(\cdot, \tau)\|_{p, \infty} \sup _{t>0}\|v(t, \cdot)\|_{2, \infty} \\
& (t-\tau)^{-1 / 2-(p-1) / p+(p-2) / 2 p}\|\varphi\|_{p /(p-1), 1} d \tau \\
\leq & C \int_{0}^{t / 2}\|w\|_{\mathscr{Y}} \sup _{t>0}\|v(t, \cdot)\|_{2, \infty} \tau^{1 / p-1 / 2}(t-\tau)^{-1} d \tau \\
\leq & C t^{1 / p-1 / 2}\|w\|_{\mathscr{Y}} \sup _{t>0}\|v(t, \cdot)\|_{2, \infty}
\end{aligned}
$$

by the standard $L^{p}-L^{q}$ estimate and real interpolation. The term $I_{3}$ can be estimated as

$$
\begin{aligned}
I_{3} & \leq C \sup _{\varphi} \int_{t / 2}^{t}\|w(\cdot, \tau)\|_{p, \infty} \sup _{t>0}\|v(t, \cdot)\|_{2, \infty}\|\nabla \exp ((t-\tau) \Delta) \varphi\|_{2 p /(p-2), 1} d \tau \\
& \leq C \sup _{\varphi} \int_{t / 2}^{t} \tau^{1 / p-1 / 2}\|w\| \mathscr{Y} \sup _{t>0}\|v(t, \cdot)\|_{2, \infty}\|\nabla \exp ((t-\tau) \Delta) \varphi\|_{2 p /(p-2), 1} d \tau \\
& \leq C t^{1 / p-1 / 2}\|w\| \mathscr{Y} \sup _{t>0}\|v(t, \cdot)\|_{2, \infty} \sup _{\varphi} \int_{0}^{t / 2}\|\nabla \exp (t \Delta) \varphi\|_{2 p /(p-2), 1} d \tau .
\end{aligned}
$$


The standard $L^{p}-L^{q}$ estimate and real interpolation imply that

$$
\begin{aligned}
& \|\nabla \exp (t \Delta) \varphi\|_{2 p /(p-2), 1} \\
& \quad \leq C t^{-1 / 2-(2 p-1) / 2 p+(p-2) / 2 p}\|\varphi\|_{2 p /(2 p-1), 1}=C t^{-1-1 / 2 p}\|\varphi\|_{2 p /(2 p-1), 1}
\end{aligned}
$$

and

$$
\begin{aligned}
& \|\nabla \exp (t \Delta) \varphi\|_{2 p /(p-2), 1} \\
& \quad \leq C t^{-1 / 2-(2 p-3) / 2 p+(p-2) / 2 p}\|\varphi\|_{2 p /(2 p-3), 1}=C t^{-1+1 / 2 p}\|\varphi\|_{2 p /(2 p-3), 1} .
\end{aligned}
$$

These estimates imply that the mapping $\varphi \mapsto\|\nabla \exp (t \Delta) \varphi\|_{2 p /(p-2), 1}$ is bounded from $L^{2 p /(2 p-1), 1}\left(\mathbb{R}^{2}\right)$ to $L^{2 p /(2 p+1), \infty}((0, \infty))$, and from $L^{2 p /(2 p-3), 1}\left(\mathbb{R}^{2}\right)$ to $L^{2 p /(2 p-1), \infty}$ $((0, \infty))$. It follows that this mapping is bounded from $\left(L^{2 p /(2 p-1), 1}\left(\mathbb{R}^{2}\right), L^{2 p /(2 p-3), 1}\right.$ $\left.\left(\mathbb{R}^{2}\right)\right)_{1 / 2,1}=L^{p /(p-1), 1}\left(\mathbb{R}^{2}\right)$ to

$$
\left(L^{2 p /(2 p+1), \infty}((0, \infty)), L^{2 p /(2 p-1), \infty}((0, \infty))\right)_{1 / 2,1}=L^{1,1}((0, \infty))=L^{1}((0, \infty)) .
$$

This implies that $\sup _{\varphi} \int_{0}^{t / 2}\|\nabla \exp (t \Delta) \varphi\|_{2 p /(p-2), 1} d \tau$ is bounded by a constant depending only on $p$. Substituting this fact into (4.9), we obtain $I_{3} \leq C t^{1 / p-1 / 2}\|w\|_{\mathscr{Y}} \sup _{t>0}\|v(t, \cdot)\|_{2, \infty}$. Form (4.7), (4.8) and this estimate we see that

$$
t^{1 / 2-1 / p}\left\|S_{0}[w](\cdot, t)\right\|_{p, \infty} \leq C\left(\|w\|_{\mathscr{Y}}^{2}+\|w\|_{\mathscr{Y}} \sup _{t>0}\|v(\cdot, t)\|_{2, \infty}\right) .
$$

From this estimate and (4.4), (4.6) we conclude that

$$
\begin{aligned}
& \left\|S\left[w_{0}, w\right](\cdot, t)\right\|_{2, \infty}+t^{1 / 2-1 / p}\left\|S\left[w_{0}, w\right](\cdot, t)\right\|_{p, \infty} \\
& \quad \leq C\left(\left\|w_{1}\right\|_{\mathscr{Y}}+\left\|w_{2}\right\|_{\mathscr{Y}}+\sup _{t>0}\|v(\cdot, t)\|_{2, \infty}\right)\|w\|_{\mathscr{Y}}+C\left\|w_{0}\right\|_{2, \infty} .
\end{aligned}
$$

This completes the proof.

\section{Declarations}

Conflict of interest The author declares that he has no conflict of interest.

Open Access This article is licensed under a Creative Commons Attribution 4.0 International License, which permits use, sharing, adaptation, distribution and reproduction in any medium or format, as long as you give appropriate credit to the original author(s) and the source, provide a link to the Creative Commons licence, and indicate if changes were made. The images or other third party material in this article are included in the article's Creative Commons licence, unless indicated otherwise in a credit line to the material. If material is not included in the article's Creative Commons licence and your intended use is not permitted by statutory regulation or exceeds the permitted use, you will need to obtain permission directly from the copyright holder. To view a copy of this licence, visit http://creativecommons.org/licenses/by/4.0/.

\section{References}

1. Bergh, J., Löfström, J.: Interpolation Spaces. Springer, Berlin (1976) 
2. Brandolese, L.: Space-time decay of Navier-Stokes flows invariant under rotations. Math. Ann. 329, 685-706 (2004)

3. Crispo, F., Maremonti, P.: Navier-Stokes equations in aperture domains: global existence with bounded flux and time-periodic solutions. Math. Methods Appl. Sci. 31, 249-277 (2008)

4. Farwig, R., Nakatsuka, T., Taniuchi, Y.: Existence of solutions on the whole time axis to the Navier-Stokes equations with precompact range in $L^{3}$. Arch. Math. 104, 539-550 (2015)

5. Farwig, R., Okabe, T.: Periodic solutions of the Navier-Stokes equations with in homogeous boundary conditions. Ann. Univ. Ferrara 56, 249-281 (2010)

6. Galdi, G.P.: Existence and uniqueness of time-periodic solutions to the Navier-Stokes equations in the whole plane. Discrete Contin. Dyn. Syst. Ser. S 6, 1237-1257 (2013)

7. Galdi, G.P.: On time-periodic flow of a viscous liquid past a moving cylinder. Arch. Rational Mech. Anal. 210, 451-498 (2013)

8. Galdi, G.P., Silvestre, A.L.: Existence of time-periodic solutions to the Navier-Stokes equations around a moving body. Pacific J. Math 223, 251-267 (2006)

9. Galdi, G.P., Silvestre, A.L.: On the motion of a rigid body in a Navier-Stokes liquid under the action of a time-periodic force. Indiana Univ. Math. J. 58, 2805-2842 (2009)

10. Galdi, G.P., Sohr, H.: Existence and uniqueness of time-periodic physically reasonable Navier-Stokes flow past a body. Arch. Ration. Mech. Anal. 172, 363-406 (2004)

11. Iwabuchi, T., Takada, R.: Time periodic solutions to the Navier-Stokes equations in the rotational framework. J. Evol. Equ. 12, 985-1000 (2012)

12. Kobayashi, T.: The motions between stationary and periodic solutions to the Navier-Stokes equations in two or three dimensional channels. J. Math. Kyoto Univ. 49, 307-323 (2009)

13. Kozono, H., Mashiko, Y., Takada, R.: Existence of periodic solutions and their asymptotic stability to the Navier-Stokes equations with the Coriolis force. J. Evol. Equ. 14, 565-601 (2014)

14. Kozono, H., Nakao, M.: Periodic solutions of the Navier-Stokes equations in unbounded domains. ibid. 48, 33-50 (1996)

15. Kubo, T.: Periodic solutions of the Navier-Stokes equations in a perturbed half-space and an aperture domain. Math. Methods Appl. Sci. 28, 1341-1357 (2005)

16. Kyed, M.: The existence and regularity of time-periodic solutions to the three-dimensional Navier-Stokes equations on the whole space. Nonlinearity 27, 2909-2935 (2014)

17. Lemarié-Rieusset, P.G.: On some classes of time-periodic solutions for the Navier-Stokes equations in the whole space. SIAM J. Math. Anal. 47, 1022-1043 (2015)

18. Maremonti, P.: Existence and stability of time periodic solution of the Navier-Stokes equations in the whole space. Nonlinearity 4, 503-529 (1991)

19. Maremonti, P.: Some theorems of existence for solutions of the Navier-Stokes equations with slip boundary conditions in half-space. Ricerche Mat. 40, 81-135 (1991)

20. Maremonti, P., Padula, M.: Existence, uniqueness and attainability of periodic solutions of the NavierStokes equations in exterior domains. Zap. Nauchn. Sem. S.-Petersburg. Otdel. Mat. Inst. Steklov. 233, 142-182 (1996); Engl. transl., J. Math. Sci. 93, 719-746 (1999)

21. Miyakawa, T.: Hardy spaces of solenoidal vector fields with applications to the Navier-Stokes equations. Kyushu J. Math. 50, 1-64 (1996)

22. Miyakawa, T.: Application of Hardy space techniques to the time-decay problems for incompressible Navier-Stokes flows in $\mathbf{R}^{n}$. Funkcialaj Ekvacioj 41, 383-434 (1998)

23. Nguyen, T.H.: Periodic motions of stokes and Navier-Stokes flows around a rotating obstacle. Arch. Rational Mech. Anal. 213, 689-703 (2014)

24. Okabe, T., Tsutsui, Y.: Navier-Stokes flow in the weighted Hardy space with applications to time decay problem. J. Differ. Equ. 261, 1712-1755 (2016)

25. Salvi, R.: On the existence of periodic weak solutions on the Navier-Stokes equations in exterior regions with periodically moving boundaries. In: Sequeira A (ed.), Navier-Stokes Equations and Related Nonlinear Problems, Proceedings 3rd International Conference in Funchal, Madeira, Portugal, 1994, Plenum, New York, pp. 63-73 (1995)

26. Silvestre, A.L.: Existence and uniqueness of time-periodic solutions with finite kinetic energy for the Navier-Stokes equations in $\mathbb{R}^{3}$. Nonlinearity 25, 37-55 (2012)

27. Yamazaki, M.: The Navier-Stokes equations in the weak- $L^{n}$ space with time-dependent external force. Math. Ann. 317, 635-675 (2000)

28. Yamazaki, M.: The stationary Navier-Stokes equation on the whole plane with external force with antisymmetry. Ann. Univ. Ferrara 55, 407-423 (2009)

29. Yamazaki, M.: Two-dimensional stationary Navier-Stokes equations with 4-cyclic symmetry. Math. Nachr. 289, 2281-2311 (2016) 